ALEA, Lat. Am. J. Probab. Math. Stat. 16, 1165-1199 (2019)

DOI: 10.30757/ALEA.v16-44

\title{
Random cover times using the Poisson cylinder process
}

\section{Erik I. Broman and Filipe Mussini}

Chalmers University of Technology and Gothenburg University

Chalmers tvärgata 3 ,

MV-huset

Gothenburg, Sweden.

E-mail address: broman@chalmers.se

Uppsala University

Lägerhyddsvägen 1,

Ångströmlaboratoriet

Uppsala, Sweden.

E-mail address: filipe.mussini@math.uu.se

\begin{abstract}
In this paper we deal with the classical problem of random cover times. We investigate the distribution of the time it takes for a Poisson process of cylinders to cover a set $A \subset \mathbb{R}^{d}$. This Poisson process of cylinders is invariant under rotations, reflections and translations, and in addition we add a time component so that cylinders are "raining from the sky" at unit rate. Our main results concerns the asymptotic of this cover time as the set $A$ grows. If the set $A$ is discrete and well separated, we show convergence of the cover time to a Gumbel distribution. If instead $A$ has positive box dimension (and satisfies a weak additional assumption), we find the correct rate of convergence.
\end{abstract}

\section{Introduction}

Many variants of coverage problems have been studied in the probabilistic literature. One of the first papers on this subject was by Dvoretzky (1956) and dealt with the problem of covering the circle by using a sequence of sets placed randomly around the circle. The related problem of covering $\mathbb{R}^{d}$ was then later studied by Shepp (1972) for $d=1$, Biermé and Estrade (2012) for general $d$ and also by Broman et al. (2017) for general $d$. A common feature of these papers was that no time-component was involved. Instead, (infinite) measures $\mu$ on the set of compact

Received by the editors January 3rd, 2019; accepted July 18th, 2019.

2010 Mathematics Subject Classification. 60K35.

Key words and phrases. Cover times, Poisson cylinder process.

Erik I. Broman would like to thank the Swedish research council (Vetenskapsrådet) for financial support. 
subsets of $\mathbb{R}^{d}$ were considered. The papers studied a Poisson process using $\mu$ as the intensity measure, and asked whether $\mathbb{R}^{d}$ would be completely covered. Of course, this depends on the particular choice of $\mu$ and this dependence was investigated.

A variant of this covering problem is to do the following. Start with some bounded set $A \subset \mathbb{R}^{d}$, and throw down other (possibly random) sets $\left(B_{i}\right)_{i \geq 1}$ sequentially and at random locations, and proceed until $A$ is covered. It is then natural to ask about the distribution of the number of sets needed to cover $A$. Alternatively, if the sets are dropped at unit rate, one can ask about the distribution of the cover time, i.e. the time it takes until $A$ is covered. An example would be to let $A$ be the unit square in $\mathbb{R}^{2}$, and to let $\left(B_{i}\right)_{i \geq 1}$ be a collection of squares of side length $\epsilon$ with their centres uniformly distributed in $A$. Another example would be to let the side length of the sets $\left(B_{i}\right)_{i>1}$ be random. Such problems were studied by for instance Siegel and Holst (1982) and Janson (1983) on the circle, while a much more general result was later obtained by Janson (1986). In particular, all of these papers studied asymptotics of the cover times as the set $A$ grew.

More recently, Belius (2012) studied the problem of covering a bounded set $A \subset \mathbb{Z}^{d}$ by what is known as random interlacements. This is basically a Poisson process on the trajectories of bi-infinite random walks in $\mathbb{Z}^{d}$ and was introduced by Sznitman (2010). The major difference between the paper by Belius and the others mentioned above, is that the interlacement trajectories are unbounded objects, whereas in the classical setting, the corresponding sets are finite. The use of infinite objects introduces a number of new challenges as the cover times of separated sets no longer are independent.

The aim of this paper is to study the classical problem of covering sets $A \subset \mathbb{R}^{d}$ of non-zero dimension (rather than say a subset of $\mathbb{Z}^{d}$ ), combined with the use of unbounded objects to cover the set. In order to explain our main result, we will give informal descriptions of the mathematical quantities and tools needed. Precise definitions and explanations will be detailed in Section 2. For the models to make sense, we will assume throughout the paper that the dimension $d$ is at least 2 .

We will use a Poisson process $\Psi$ where an element $(L, s) \in \Psi$ consists of a line $L \subset \mathbb{R}^{d}$ and a "time-stamp" $s \in \mathbb{R}^{+}$. The set of lines with time-stamp smaller than $t$ will then be a Poisson process on the set of lines in $\mathbb{R}^{d}$, and this process will be invariant under rotations, reflections and translations (see Section 2). For such a line $L$ we consider the corresponding cylinder $\mathfrak{c}(L)$ with base radius 1 , i.e.

$$
\mathfrak{c}(L):=\left\{x \in \mathbb{R}^{d}: d(x, L) \leq 1\right\} .
$$

We then define the cover time of $A$ to be

$$
\mathcal{T}(A):=\inf \left\{t>0: A \subset \bigcup_{(L, s) \in \Psi: s \leq t} \mathfrak{c}(L)\right\},
$$

so that $\mathcal{T}(A)$ is the first time when $A$ has been covered by the cylinders.

In order to state our main result, we will need to define the box dimension of a bounded set $A \subset \mathbb{R}^{d}$. A further (albeit brief) discussion of this concept is included in Section 2.2. Let $N_{\delta}(A)$ be the minimum number of boxes of side length $\delta>0$ needed to cover $A$. The box dimension of $A$ is then defined by

$$
\operatorname{dim}_{B}(A)=\lim _{\delta \rightarrow 0} \frac{\log N_{\delta}(A)}{-\log \delta}
$$


whenever this limit exists.

We can now state our main theorem.

Theorem 1.1. For any set $A \subset \mathbb{R}^{d}$ such that $\operatorname{dim}_{B}(A)$ is well defined, and which satisfies the additional condition

$$
0<\liminf _{\delta \rightarrow 0} \delta^{\operatorname{dim}_{B}(A)} N_{\delta}(A) \leq \limsup _{\delta \rightarrow 0} \delta^{\operatorname{dim}_{B}(A)} N_{\delta}(A)<\infty,
$$

we have that the sequence

$$
\left(\mathcal{T}(n A)-\operatorname{dim}_{B}(A)(\log n+\log \log n)\right)_{n \geq 1}
$$

is tight.

Remarks: $n A$ is simply the set $A$ scaled by a factor of $n$.

It is reasonable (in light of similar results such as the ones in Janson, 1986), to expect that if $A$ satisfies a strong enough regularity condition,

$$
\mathcal{T}(n A)-\operatorname{dim}_{B}(A)(\log n+\log \log n)+C
$$

converges to a Gumbel distribution for a suitable choice of constant $C$. Our main result does not quite achieve this. However, it does show that if $\mathcal{T}(n A)-f(n)$ converges to a non-trivial random variable, then the function $f(n)$ must in fact take the form $\operatorname{dim}_{B}(A)(\log n+\log \log n)+O(1)$. See also the remark after the proof of Theorem 1.1.

It might not be clear whether a "typical" set should satisfy condition (1.1). While we will not discuss this question in full detail (as it is more a statement that belongs to fractal geometry), we give a basic result (Proposition 8.1) and provide a few examples in Section 8. That section provides sufficient motivation that assumption (1.1) is satisfied for a rich family of sets.

In Theorem 2.1, we will provide an auxiliary (and somewhat weaker) result that covers the case when (1.1) fails.

Our second main result deals with sequences of finite subsets of $\mathbb{R}^{d}$. As this text considers both finite subsets of $\mathbb{R}^{d}$ and non-discrete subsets, it will be convenient to use different notations for the two. Therefore we let $\mathbb{A}, \mathbb{D}$ etc denote finite subsets of $\mathbb{R}^{d}$, while $A \subset \mathbb{R}^{d}$ will be used to denote a non-discrete set. We let $d(x, y)$ denote the regular Euclidean distance between $x, y \in \mathbb{R}^{d}$, and we will use the following definition

$$
\operatorname{Sep}(\mathbb{D}):=\inf \{d(x, y): x, y \in \mathbb{D}\},
$$

so that $\operatorname{Sep}(\mathbb{D})$ denotes the minimum distance between any two points in the set $\mathbb{D}$. A finite set $\mathbb{D}$ with $\operatorname{Sep}(\mathbb{D}) \geq \rho$ will be called $\rho$-separated.

Here and in the rest of the paper, $|\cdot|$ denotes cardinality.

Theorem 1.2. Let $\left(\mathbb{D}_{n}\right)_{n \geq 1}$ be a sequence of finite subsets of $\mathbb{R}^{d}$. If $\lim _{n \rightarrow \infty}\left|\mathbb{D}_{n}\right|=$ $\infty$ and

$$
\liminf _{n} \operatorname{Sep}\left(\mathbb{D}_{n}\right) \log \left|\mathbb{D}_{n}\right|=\infty,
$$

then $\left(\mathcal{T}\left(\mathbb{D}_{n}\right)-\log \left|\mathbb{D}_{n}\right|\right)_{n \geq 1}$ converges in distribution to the Gumbel distribution as $n \rightarrow \infty$.

Remark: In Section 5, we will prove Theorem 5.7, which provides a bound for the fluctuation of $\mathbb{P}(\mathcal{T}(\mathbb{D})-\log |\mathbb{D}| \leq z)$ from the Gumbel distribution function. This theorem will then readily imply Theorem 1.2. 
The same fluctuation result will also be used later to prove Theorem 1.1. In order to give an informal explanation of how this is done, consider Theorem 1.1 and some set $A$. We will consider a carefully chosen finite set sitting inside of $n A$, and the cover time of this set will provide a lower bound to $\mathcal{T}(n A)$. In order to find an upper bound on $\mathcal{T}(n A)$, we will consider the same discrete set, but require that each point is singularly covered (meaning that a small ball around the point is covered by a single cylinder of the process). If all points are singularly covered, this will imply that the set $n A$ is covered, and this allows us to obtain an upper bound on the cover time of $n A$.

Before we wrap up this section, we want to provide a short intuitive explanation of the overall strategy of proving our main results. Informally, if the cover time is $\tau$, then immediately before this time (i.e. at time $\tau(1-\epsilon)$ ), the set which is uncovered will, with high probability, consist of small islands which are well separated. Indeed, this is the case as Proposition 5.5 shows. In addition, Proposition 5.5 tells us that the number of such islands will be highly concentrated around its expected value. This can then be combined with Proposition 5.1 which tells us that the cover times of these well separated islands are almost independent. These two results are then combined into Theorem 5.7, which provides the final estimate on the fluctuations of the cover time $\mathcal{T}(\mathbb{D})$. This result is then in turn used to prove both Theorem 1.1 and Theorem 1.2.

The structure of the rest of the paper is as follows: In Section 2 we define the Poisson cylinder model and briefly discuss box dimensions. In Section 3 we present some preliminary results concerning the Poisson cylinder model, while in Section 4 we prove some fundamental properties about $\rho$-separated sets. Then, Section 5 is devoted to the proof of the fluctuation result, i.e. Theorem 5.7 which is used to prove Theorem 1.2 in the same section. The overall strategy in this section is similar to the strategy of Belius (2012) and described above. However, there will also be many differences stemming from the fact that we are working in $\mathbb{R}^{d}$ as opposed to the discrete space $\mathbb{Z}^{d}$. Section 6 is dedicated to proving Theorem 1.1 while Section 7 is used to prove our above mentioned secondary result, i.e. Theorem 2.1. Finally, Section 8 contains some examples and Proposition 8.1 that pertains to assumption (1.1) of Theorem 1.1.

We end this section with a comment on notation. We shall frequently use $c$ to denote a constant (depending only on $d$ ) which may change from line to line. In contrast, numbered constants $c_{k}$ will be fixed.

\section{Model and definitions}

This section is divided into two subsections. The first one includes the definition and some preliminaries of the Poisson cylinder model. The second subsection provides some basic background on box dimensions.

2.1. The Poisson cylinder model. Our first step is to define the Poisson line model. To that end, let $G(d, 1)$ be the set of infinite lines in $\mathbb{R}^{d}$ that pass through the origin $o$, and let $A(d, 1)$ be the set of infinite lines in $\mathbb{R}^{d}$. Furthermore, let

$$
\mathcal{L}_{K}:=\{L \in A(d, 1): L \cap K \neq \emptyset\},
$$


be the set of lines that intersect $K$ where $K \subset \mathbb{R}^{d}$ is a compact set. For convenience, we let $\mathcal{L}_{A, B}$ denote the set $\mathcal{L}_{A} \cap \mathcal{L}_{B}$, i.e. the set of lines that intersect both sets $A, B \subset \mathbb{R}^{d}$. Furthermore, $B(x, \rho)$ will denote the closed ( $d$-dimensional) ball of radius $\rho$ centered at $x$.

We let $\nu_{d, 1}$ be the unique Haar measure on the space $G(d, 1)$ normalized so that $\nu_{d, 1}(G(d, 1))=1$. Furthermore, on $A(d, 1)$ there is a unique (up to constants) measure which is invariant under rotations, reflections and translations. We will let $\mu_{d, 1}$ denote this latter measure, normalized so that $\mu_{d, 1}\left(\mathcal{L}_{B(o, 1)}\right)=1$ (see for instance Schneider and Weil, 2008 Chapter 13). For any subspace $H \subset \mathbb{R}^{d}$, and set $D \subset \mathbb{R}^{d}$, we let $\Pi_{H}(D)$ denote the projection of $D$ onto $H$. Here, we will consider $\Pi_{H}(D)$ to be a subset of $\mathbb{R}^{d}$ (and not just a subset of $H$ ). Furthermore, we will let $\kappa_{d}$ denote the volume of the unit ball $B(o, 1)$ in $\mathbb{R}^{d}$, and $\lambda_{d}$ denote Lebesgue measure on $\mathbb{R}^{d}$ so that $\kappa_{d}=\lambda_{d}(B(o, 1))$.

For any $L \in G(d, 1)$ we will let $L^{\perp}$ be the $(d-1)$-dimensional hyperplane orthogonal to $L$ and containing the origin $o$. The following representation (Schneider and Weil (2008) Theorem 13.2.12) of the measure $\mu_{d, 1}$ will be useful for us. For any $K \subset \mathbb{R}^{d}$ we have that

$$
\mu_{d, 1}\left(\mathcal{L}_{K}\right)=\frac{1}{\kappa_{d-1}} \int_{G(d, 1)} \int_{L^{\perp}} \mathbb{1}\left(L+y \in \mathcal{L}_{K}\right) \lambda_{d-1}(\mathrm{~d} y) \nu_{d, 1}(\mathrm{~d} L),
$$

where $\mathbb{1}$ denotes an indicator function. Informally, for a fixed line $L$, the inner integral integrates over all lines parallel to $L$ that intersect $K$. Then, the outer integral integrates over all possible choices of $L$.

Our next step is to consider the following space of point measures on $A(d, 1)$,

$$
\Omega=\left\{\omega=\sum_{i=1}^{\infty} \delta_{L_{i}}: L_{i} \in A(d, 1) \text { and } \omega\left(\mathcal{L}_{A}\right)<\infty \text { for all compact } A \subset \mathbb{R}^{d}\right\},
$$

where $\delta_{L}$ denotes point measure at $L$. By standard abuse of notation, we will sometimes identify the random measure $\omega \in \Omega$ with its $\operatorname{support} \operatorname{supp}(\omega)$, which is really a subset of $A(d, 1)$.

We define $\Psi$ to be a Poisson point process on $A(d, 1) \times \mathbb{R}^{+}$with intensity measure $\mu_{d, 1} \times \lambda_{1}^{+}$where $\lambda_{1}^{+}$denotes Lebesgue measure on $\mathbb{R}^{+}$. We then think of an element $(L, s) \in \Psi$ as a line in $\mathbb{R}^{d}$ accompanied with a time-stamp $s$. We then let $\omega_{t}=$ $\Pi_{A(d, 1)}\{(L, s) \in \Psi: s \leq t\}$, where $\Pi_{A(d, 1)}$ denotes projection onto the space $A(d, 1)$. Thus, $\omega_{t}$ is the collection of lines which have been placed before or at time $t$, and $\omega_{t} \in \Omega$. By our definition, $\omega_{t}$ is in fact a Poisson process on $A(d, 1)$ with intensity measure $t \mu_{d, 1}$. Similarly, we let $\omega_{t_{1}, t_{2}}=\Pi_{A(d, 1)}\left\{(L, s) \in \Psi: t_{1}<s \leq t_{2}\right\}$, so that $\omega_{t_{1}, t_{2}}$ is the set of lines placed between times $t_{1}$ and $t_{2}$. The intensity measure of $\omega_{t_{1}, t_{2}}$ is therefore $\left(t_{2}-t_{1}\right) \mu_{d, 1}$. Obviously we have that $\omega_{t_{1}} \cup \omega_{t_{1}, t_{2}}=\omega_{t_{2}}$, and we will let $\left(\omega_{t}\right)_{t \geq 0}$ denote the corresponding process.

We will sometimes slightly abuse notation by writing $\mathfrak{c}(L) \in \omega_{t}$ instead of $L \in \omega_{t}$, and we will often think of (and refer to) $\omega_{t}$ as a collection of cylinders instead of lines.

2.2. Box dimensions. In this subsection we will review some basic properties of box dimensions, sometimes referred to as Minkowski dimensions (see Falconer, 2014 Chapter 3). 
Recall the definition of $N_{\delta}(A)$ from the introduction. Then, define

$$
\overline{\operatorname{dim}}_{B}(A):=\limsup _{\delta \rightarrow 0} \frac{\log N_{\delta}(A)}{-\log \delta},
$$

called the upper box dimension of the set $A$. Similarly, we define

$$
\underline{\operatorname{dim}}_{B}(A):=\liminf _{\delta \rightarrow 0} \frac{\log N_{\delta}(A)}{-\log \delta}
$$

to be the lower box dimension of the set $A$, and if these coincide, then

$$
\operatorname{dim}_{B}(A)=\overline{\operatorname{dim}}_{B}(A)=\underline{\operatorname{dim}}_{B}(A)
$$

is simply called the box dimension of $A$.

The two quantities in (1.1) are related to the upper and lower Minkowski content (see Federer, 1969, Sections 3.2.37-3.2.44). It is beyond the scope of this paper to investigate this relationship in detail. However, as mentioned already in the remarks after the statement of Theorem 1.1, it will be the case that (1.1) is satisfied for many sets. See in particular Proposition 8.1.

We can now present Theorem 2.1 mentioned in the remarks after Theorem 1.1.

Theorem 2.1. For any $\underline{\alpha}<\underline{\operatorname{dim}}_{B}(A)$, we have that for every $z \in \mathbb{R}$,

$$
\lim _{n \rightarrow \infty} \mathbb{P}(\mathcal{T}(n A)-\underline{\alpha} \log n \leq z)=0 .
$$

Furthermore, for any $\bar{\alpha}>\overline{\operatorname{dim}}_{B}(A)$, we have that for every $z \in \mathbb{R}$,

$$
\lim _{n \rightarrow \infty} \mathbb{P}(\mathcal{T}(n A)-\bar{\alpha} \log n \leq z)=1 .
$$

Remark: One can consider the cover times along a subsequence $\left(n_{k}\right)_{k \geq 1}$ such that

$$
\lim _{k \rightarrow \infty} \frac{\log N_{1 / n_{k}}(A)}{\log n_{k}}=\overline{\operatorname{dim}}_{B}(A) .
$$

If in addition,

$$
0<\liminf _{k \rightarrow \infty} n_{k}^{-\overline{\operatorname{dim}}_{B}(A)} N_{1 / n_{k}}(A) \leq \limsup _{k \rightarrow \infty} n_{k}^{-\overline{\operatorname{dim}}_{B}(A)} N_{1 / n_{k}}(A)<\infty,
$$

then, one will obtain a result resembling Theorem 1.1 but along this subsequence, and with $\overline{\operatorname{dim}}_{B}(A)$ in place of $\operatorname{dim}_{B}(A)$. We also see that Theorem 2.1 covers the cases when

$$
\liminf _{\delta \rightarrow 0} \delta^{\operatorname{dim}_{B}(A)} N_{\delta}(A)=0 \text { or } \limsup _{\delta \rightarrow 0} \delta^{\operatorname{dim}_{B}(A)} N_{\delta}(A)=\infty .
$$

That is, for those sets $A \subset \mathbb{R}^{d}$ with a well defined box dimension but where assumption (1.1) fails.

\section{Preliminary results concerning the Poisson cylinder model}

The next result allows us to estimate the measure of the set of cylinders intersecting two distant balls. This lemma was first published as Lemma 3.1 of Tykesson and Windisch (2012). Here, we present a sketch for sake of completeness.

Lemma 3.1. Let $x_{1}, x_{2} \in \mathbb{R}^{d}$ and let $r=d\left(x_{1}, x_{2}\right)$. There exist constants $c_{1}$ and $c_{2}$ depending only on $d$ such that

$$
\frac{c_{1}}{r^{d-1}} \leq \mu_{d, 1}\left(\mathcal{L}_{B\left(x_{1}, 1\right), B\left(x_{2}, 1\right)}\right) \leq \frac{c_{2}}{r^{d-1}},
$$

for every $r \geq 4$. 
Sketch of proof. By translation invariance of $\mu_{d, 1}$, we can, without loss of generality assume that $x_{1}=o$ so that $x_{2}$ is located on the surface of $B(o, r)$. Furthermore, we need order $r^{(d-1)}$ balls of radius 1 to cover the surface of $B(o, r)$. By symmetry, a random line passing through $B(o, 1)$ will hit any fixed ball in the cover with equal probability. Thus, the probability that it will hit $B\left(x_{2}, 1\right)$ must be of order $r^{-(d-1)}$.

The next lemma states that, despite the long-range correlation nature of the Poisson cylinder process, events occurring in two distant sets are almost independent. The key point of the proof is to note that the events become independent after we conditioned on the event that no lines intersect both sets.

Lemma 3.2. Let $K_{1}, K_{2} \subset \mathbb{R}^{d}$ be disjoint sets and let $E_{1}$ and $E_{2}$ be events depending only on $\omega_{t}$ in $\mathcal{L}_{K_{1}}$ and $\mathcal{L}_{K_{2}}$ respectively. Then:

$$
\left|\mathbb{P}\left(E_{1} \cap E_{2}\right)-\mathbb{P}\left(E_{1}\right) \mathbb{P}\left(E_{2}\right)\right| \leq 4 \mathbb{P}\left(\omega_{t}\left(\mathcal{L}_{K_{1}, K_{2}}\right) \neq 0\right) .
$$

Proof. Note that

$$
\begin{aligned}
\mathbb{P}\left(E_{1}\right. & \left.\cap E_{2}\right) \\
= & \mathbb{P}\left(E_{1} \mid \omega_{t}\left(\mathcal{L}_{K_{1}, K_{2}}\right)=0\right) \mathbb{P}\left(E_{2} \mid \omega_{t}\left(\mathcal{L}_{K_{1}, K_{2}}\right)=0\right) \mathbb{P}\left(\omega_{t}\left(\mathcal{L}_{K_{1}, K_{2}}\right)=0\right) \\
& +\mathbb{P}\left(E_{1} \cap E_{2} \mid \omega_{t}\left(\mathcal{L}_{K_{1}, K_{2}}\right) \neq 0\right) \mathbb{P}\left(\omega_{t}\left(\mathcal{L}_{K_{1}, K_{2}}\right) \neq 0\right),
\end{aligned}
$$

since the events $E_{1}$ and $E_{2}$ are conditionally independent on $\omega_{t}\left(\mathcal{L}_{K_{1}, K_{2}}\right)=0$. Furthermore, writing

$$
\begin{aligned}
\mathbb{P}\left(E_{i}\right) & =\mathbb{P}\left(E_{i} \mid \omega_{t}\left(\mathcal{L}_{K_{1}, K_{2}}\right)=0\right) \mathbb{P}\left(\omega_{t}\left(\mathcal{L}_{K_{1}, K_{2}}\right)=0\right) \\
& +\mathbb{P}\left(E_{i} \mid \omega_{t}\left(\mathcal{L}_{K_{1}, K_{2}}\right) \neq 0\right) \mathbb{P}\left(\omega_{t}\left(\mathcal{L}_{K_{1}, K_{2}}\right) \neq 0\right)
\end{aligned}
$$

for $i=1,2$ and using (3.1), a straightforward calculation gives us that

$$
\left|\mathbb{P}\left(E_{1} \cap E_{2}\right)-\mathbb{P}\left(E_{1}\right) \mathbb{P}\left(E_{2}\right)\right| \leq 4 \mathbb{P}\left(\omega_{t}\left(\mathcal{L}_{K_{1}, K_{2}}\right) \neq 0\right)
$$

as desired.

As explained in the end of the introduction, one step in proving the main theorems is to show that the cover times of distant and small sets $K_{1}, \ldots, K_{n+1}$ are almost independent. If we consider one of these sets $K_{j}$, the next lemma gives us bounds on the probability that there exists a line passing through $K_{j}$ and any one of the other sets $K_{i}, i \neq j$.

Lemma 3.3. Let $\left\{K_{i}\right\}_{i=1}^{n+1}$ be a family of sets such that for every $i, K_{i} \subset B\left(x_{i}, 1\right)$ for some $x_{i} \in \mathbb{R}^{d}$. Assume also that $d\left(x_{i}, x_{j}\right)=r_{i j} \geq 4$ for every $i \neq j$ and let $r=\min _{i \neq j} r_{i j}$. Then, for all $1 \leq j \leq n+1$ we have

$$
\mathbb{P}\left(\omega_{t}\left(\mathcal{L}_{\bigcup_{i=1}^{n+1} K_{i} \backslash K_{j}, K_{j}}\right) \neq 0\right) \leq \frac{n t c_{2}}{r^{d-1}},
$$

where $c_{2}$ is the same as in Lemma 3.1.

Proof. Fix $j$ and let $B=\bigcup_{i=1}^{n+1} B\left(x_{i}, 1\right)$. By Lemma 3.1 and a simple union bound we have that for any $i$,

$$
\mu_{d, 1}\left(\mathcal{L}_{\left(B \backslash B\left(x_{i}, 1\right)\right), B\left(x_{i}, 1\right)}\right) \leq n c_{2} \frac{1}{r^{d-1}} .
$$


Therefore we get that

$$
\begin{aligned}
& \mathbb{P}\left(\omega_{t}\left(\mathcal{L}_{\bigcup_{i=1}^{n+1} K_{i} \backslash K_{j}, K_{j}}\right) \neq 0\right) \leq \mathbb{P}\left(\omega_{t}\left(\mathcal{L}_{B \backslash B\left(x_{j}, 1\right), B\left(x_{j}, 1\right)}\right) \neq 0\right) \\
& \quad=1-\exp \left(-t \mu_{d, 1}\left(\mathcal{L}_{B \backslash B\left(x_{j}, 1\right), B\left(x_{j}, 1\right)}\right)\right) \leq 1-\exp \left(-n t c_{2} \frac{1}{r^{d-1}}\right) .
\end{aligned}
$$

The result follows by using that $e^{-x} \geq 1-x$ for all $x \in \mathbb{R}$.

Lemma 3.1 applies to $\mu_{d, 1}\left(\mathcal{L}_{B(o, 1)} \cap \mathcal{L}_{B\left(r e_{1}, 1\right)}\right)$ for large values of $r$. Next, we need to consider the same expression but for small values of $r$. In contrast to Lemma 3.1, no such result exists in the literature, and so we provide a full proof.

Proposition 3.4. If $d \geq 3$ and $r \leq 2 \sqrt{1-4^{-1 /(d-2)}}$ then we have that

$$
\mu_{d, 1}\left(\mathcal{L}_{B(o, 1)} \cap \mathcal{L}_{B\left(r e_{1}, 1\right)}\right) \leq 1-\frac{r}{12} .
$$

In addition, (3.2) holds for every $r \leq 2$ when $d=2$.

Proof. Observe that for any fixed $L \in G(d, 1)$, the set of $y \in L^{\perp}$ such that $L+y \in \mathcal{L}_{K}$ is precisely $\Pi_{L^{\perp}}(K)$. From (2.1) it then follows that

$$
\begin{aligned}
& \mu_{d, 1}\left(\mathcal{L}_{B(o, 1)} \cap \mathcal{L}_{B\left(r e_{1}, 1\right)}\right) \\
&= \mu_{d, 1}\left(\mathcal{L}_{B(o, 1)}\right)+\mu_{d, 1}\left(\mathcal{L}_{B\left(r e_{1}, 1\right)}\right)-\mu_{d, 1}\left(\mathcal{L}_{B(o, 1) \cup B\left(r e_{1}, 1\right)}\right) \\
&= \frac{1}{\kappa_{d-1}} \int_{G(d, 1)} \int_{L^{\perp}} \mathbb{1}\left(L+y \in \mathcal{L}_{B(o, 1)}\right)+\mathbb{1}\left(L+y \in \mathcal{L}_{B\left(r e_{1}, 1\right)}\right) \\
& \quad-\mathbb{1}\left(L+y \in \mathcal{L}_{B(o, 1) \cup B\left(r e_{1}, 1\right)}\right) \lambda_{d-1}(\mathrm{~d} y) \nu_{d, 1}(\mathrm{~d} L) \\
&=\frac{1}{\kappa_{d-1}} \int_{G(d, 1)} \int_{L^{\perp}} \mathbb{1}\left(L+y \in \mathcal{L}_{B(o, 1)}\right) \mathbb{1}\left(L+y \in \mathcal{L}_{B\left(r e_{1}, 1\right)}\right) \lambda_{d-1}(\mathrm{~d} y) \nu_{d, 1}(\mathrm{~d} L) \\
&=\frac{1}{\kappa_{d-1}} \int_{G(d, 1)} \lambda_{d-1}\left(\Pi_{L^{\perp}}(B(o, 1)) \cap \Pi_{L^{\perp}}\left(B\left(r e_{1}, 1\right)\right)\right) \nu_{d, 1}(\mathrm{~d} L) .
\end{aligned}
$$

If $L \in G(d, 1)$ is written as $L=\left\{s\left(l_{1}, \ldots, l_{d}\right): s \in \mathbb{R}\right\}$ where $l_{1}^{2}+\ldots+l_{d}^{2}=1$, then the projection matrix $\Pi_{L^{\perp}}$ has elements $\left(\Pi_{L^{\perp}}\right)_{i i}=1-l_{i}^{2}$ and $\left(\Pi_{L^{\perp}}\right)_{i j}=-l_{i} l_{j}$ for $i \neq j$. Let $p_{r}=p_{r}(L)=\Pi_{L^{\perp}}((r, 0, \ldots, 0))$ so that $p_{r}$ is the projection of the center of $B\left(r e_{1}, 1\right)$. Of course, the projection of $B(o, 1)$ onto $L^{\perp}$ is then a $(d-1)$ dimensional ball of radius 1 centred at $o$. Straightforward calculations yield that

$$
p_{r}=r\left(1-l_{1}^{2},-l_{1} l_{2}, \ldots,-l_{1} l_{d}\right),
$$

so that

$\left|p_{r}\right|^{2}=r^{2}\left(\left(1-l_{1}^{2}\right)^{2}+l_{1}^{2} l_{2}^{2}+\cdots+l_{1}^{2} l_{d}^{2}\right)=r^{2}\left(1-2 l_{1}^{2}+l_{1}^{2}\left(l_{1}^{2}+\cdots+l_{d}^{2}\right)\right)=r^{2}\left(1-l_{1}^{2}\right)$.

Of course, $\Pi_{L^{\perp}}(B(o, 1))$ and $\Pi_{L^{\perp}}\left(B\left(r e_{1}, 1\right)\right)$ intersect whenever $\left|p_{r}\right| \leq 2$, or equivalently whenever $r^{2}\left(1-l_{1}^{2}\right) \leq 4$. Since we are assuming that $r \leq 2$, this is always satisfied. Furthermore, the $((d-1)$-dimensional $)$ volume of the lens-shaped area $\Pi_{L^{\perp}}(B(o, 1)) \cap \Pi_{L^{\perp}}\left(B\left(r e_{1}, 1\right)\right)$ is then the sum of the volumes of two spherical caps of height $h=1-\left|p_{r}\right| / 2$. The volume of one such spherical cap is (see Li (2011)) given by

$$
\frac{1}{2} \kappa_{d-1} J_{2 h-h^{2}}\left(\frac{d}{2}, \frac{1}{2}\right) .
$$


As above, $\kappa_{d-1}$ is the volume of the unit ball in $\mathbb{R}^{d-1}$ while $J_{x}(a, b)$ denotes the so-called regularized incomplete beta function defined by

$$
J_{x}(a, b)=\frac{\int_{0}^{x} t^{a-1}(1-t)^{b-1} \mathrm{~d} t}{\int_{0}^{1} t^{a-1}(1-t)^{b-1} \mathrm{~d} t} .
$$

We note that $2 h-h^{2}=1-\left|p_{r}\right|^{2} / 4=1-r^{2}\left(1-l_{1}^{2}\right) / 4$, so that (3.3) becomes

$$
\mu_{d, 1}\left(\mathcal{L}_{B(o, 1)} \cap \mathcal{L}_{B\left(r e_{1}, 1\right)}\right)=\int_{G(d, 1)} J_{1-r^{2}\left(1-l_{1}^{2}\right) / 4}\left(\frac{d}{2}, \frac{1}{2}\right) \nu_{d, 1}(\mathrm{~d} L) .
$$

Furthermore,

$$
J_{1-r^{2}\left(1-l_{1}^{2}\right) / 4}\left(\frac{d}{2}, \frac{1}{2}\right)=\frac{\int_{0}^{1-r^{2}\left(1-l_{1}^{2}\right) / 4} t^{\frac{d}{2}-1}(1-t)^{\frac{1}{2}-1} \mathrm{~d} t}{\int_{0}^{1} t^{\frac{d}{2}-1}(1-t)^{\frac{1}{2}-1} \mathrm{~d} t} .
$$

Let $D_{d}:=\int_{0}^{1} t^{\frac{d}{2}-1}(1-t)^{-\frac{1}{2}} d t$ so that

$$
J_{1-r^{2}\left(1-l_{1}^{2}\right) / 4}\left(\frac{d}{2}, \frac{1}{2}\right)=1-\frac{1}{D_{d}} \int_{1-r^{2}\left(1-l_{1}^{2}\right) / 4}^{1} t^{\frac{d}{2}-1}(1-t)^{-\frac{1}{2}} \mathrm{~d} t .
$$

Furthermore, we trivially have that (since $d \geq 2$,)

$$
D_{d}=\int_{0}^{1} t^{\frac{d}{2}-1}(1-t)^{-\frac{1}{2}} \mathrm{~d} t \leq \int_{0}^{1}(1-t)^{-1 / 2} \mathrm{~d} t=2 .
$$

We proceed to bound the integral on the right hand side of (3.5) from below. We have that

$$
\begin{aligned}
& \int_{1-r^{2}\left(1-l_{1}^{2}\right) / 4}^{1} t^{\frac{d}{2}-1}(1-t)^{-\frac{1}{2}} \mathrm{~d} t \\
& \quad \geq\left(1-r^{2}\left(1-l_{1}^{2}\right) / 4\right)^{\frac{d}{2}-1} \int_{1-r^{2}\left(1-l_{1}^{2}\right) / 4}^{1}(1-t)^{-\frac{1}{2}} \mathrm{~d} t \\
& \quad=\left(1-r^{2}\left(1-l_{1}^{2}\right) / 4\right)^{\frac{d}{2}-1} 2 \sqrt{r^{2}\left(1-l_{1}^{2}\right) / 4} \\
& \quad=\left(1-r^{2}\left(1-l_{1}^{2}\right) / 4\right)^{\frac{d}{2}-1} r \sqrt{1-l_{1}^{2}}
\end{aligned}
$$

so that by (3.4), (3.5) and (3.6) we have that

$$
\begin{aligned}
\mu_{d, 1} & \left(\mathcal{L}_{B(o, 1)} \cap \mathcal{L}_{B\left(r e_{1}, 1\right)}\right) \\
& \leq \int_{G(d, 1)} 1-\frac{1}{2}\left(\left(1-r^{2}\left(1-l_{1}^{2}\right) / 4\right)^{\frac{d}{2}-1} r \sqrt{1-l_{1}^{2}}\right) \nu_{d, 1}(\mathrm{~d} L) \\
& =1-\frac{r}{2} \int_{G(d, 1)}\left(1-r^{2}\left(1-l_{1}^{2}\right) / 4\right)^{\frac{d}{2}-1} \sqrt{1-l_{1}^{2}} \nu_{d, 1}(\mathrm{~d} L),
\end{aligned}
$$

which uses that $\nu_{d, 1}(G(d, 1))=1$ (see Section 2).

In order to estimate the right hand side of (3.7), consider the sets $G_{k}:=\{L \in$ $\left.G(d, 1): l_{k}^{2}>1 / 2\right\}$. Since $l_{1}^{2}+\cdots+l_{d}^{2}=1$, we clearly have that $G_{k} \cap G_{m}=\emptyset$ if $k \neq m$. Therefore

$$
1=\nu_{d, 1}(G(d, 1)) \geq \nu_{d, 1}\left(\cup_{k=1}^{d} G_{k}\right)=d \nu_{d, 1}\left(G_{1}\right),
$$

so that $\nu_{d, 1}\left(G_{1}\right) \leq 1 / d$. We get that

$$
\int_{G(d, 1)} \mathbb{1}\left(\left|l_{1}\right| \leq 1 / \sqrt{2}\right) \nu_{d, 1}(\mathrm{~d} L)=1-\nu_{d, 1}\left(G_{1}\right) \geq \frac{d-1}{d} .
$$


We will split (3.7) into two cases. First, consider $d=2$, so that

$$
\begin{aligned}
\mu_{2,1}\left(\mathcal{L}_{B(o, 1)} \cap \mathcal{L}_{B\left(r e_{1}, 1\right)}\right) \\
\leq 1-\frac{r}{2} \int_{G(2,1)} \sqrt{1-l_{1}^{2}} \nu_{2,1}(\mathrm{~d} L) \\
\leq 1-\frac{r}{2} \int_{G(2,1)} \sqrt{1-l_{1}^{2}} \mathbb{1}\left(\left|l_{1}\right| \leq 1 / \sqrt{2}\right) \nu_{2,1}(\mathrm{~d} L) \\
\quad \leq 1-\frac{r}{2 \sqrt{2}} \int_{G(2,1)} \mathbb{1}\left(\left|l_{1}\right| \leq 1 / \sqrt{2}\right) \nu_{2,1}(\mathrm{~d} L) \leq 1-\frac{r}{4 \sqrt{2}},
\end{aligned}
$$

where we use (3.8) in the last inequality.

Second, consider any $d \geq 3$. Since $r \leq 2 \sqrt{1-4^{-1 /(d-2)}}$ it follows that

$$
1-\frac{r^{2}}{4} \geq 2^{-2 /(d-2)} \text {. }
$$

We therefore get that

$$
\left(1-r^{2}\left(1-l_{1}^{2}\right) / 4\right)^{\frac{d}{2}-1} \geq\left(1-r^{2} / 4\right)^{\frac{d-2}{2}} \geq \frac{1}{2} .
$$

Hence, by (3.7) and the above, we conclude that

$$
\begin{aligned}
\mu_{d, 1} & \left(\mathcal{L}_{B(o, 1)} \cap \mathcal{L}_{B\left(r e_{1}, 1\right)}\right) \\
& \leq 1-\frac{r}{2} \int_{G(d, 1)}\left(1-r^{2}\left(1-l_{1}^{2}\right) / 4\right)^{\frac{d}{2}-1} \sqrt{1-l_{1}^{2}} \nu_{d, 1}(\mathrm{~d} L) \\
& \leq 1-\frac{r}{4} \int_{G(d, 1)} \sqrt{1-l_{1}^{2}} \nu_{d, 1}(\mathrm{~d} L) \\
& \leq 1-\frac{r}{8} \int_{G(d, 1)} \mathbb{1}\left(\left|l_{1}\right| \leq 1 / \sqrt{2}\right) \nu_{d, 1}(\mathrm{~d} L) \\
& \leq 1-\frac{r}{8} \frac{d-1}{d} \leq 1-\frac{r}{12},
\end{aligned}
$$

where we use (3.8) in the penultimate inequality.

Define

$$
\beta(\rho, k):=\mu_{d, 1}\left(\mathcal{L}_{B(o, 1) \cup B\left(2^{k} \rho e_{1}, 1\right)}\right)
$$

The next lemma gives both upper and lower bounds for $\beta(\rho, k)$, that will be used in the proofs of Lemma 5.4. The proof is an application of the previous proposition.

Lemma 3.5. We have that $1+\frac{2^{k}}{12} \rho<\beta(\rho, k)<2$, for all $k$ such that $2^{k} \rho \leq$ $2 \sqrt{1-4^{-1 /(d-2)}}$ for $d \geq 3$, or $2^{k} \rho \leq 2$ when $d=2$.

Proof. Clearly, $\beta(\rho, k)<2 \mu_{d, 1}\left(\mathcal{L}_{B(o, 1)}\right)=2$. For the lower bound, note that,

$$
\begin{aligned}
& \beta(\rho, k)=\mu_{d, 1}\left(\mathcal{L}_{B(o, 1)} \cup \mathcal{L}_{B\left(2^{k} \rho e_{1}, 1\right)}\right) \\
& \quad=\mu_{d, 1}\left(\mathcal{L}_{B(o, 1)}\right)+\mu_{d, 1}\left(\mathcal{L}_{B\left(2^{k} \rho e_{1}, 1\right)}\right)-\mu_{d, 1}\left(\mathcal{L}_{B(o, 1)} \cap \mathcal{L}_{B\left(2^{k} \rho e_{1}, 1\right)}\right) \\
& \geq 2-\left(1-2^{k} \frac{\rho}{12}\right)=1+\frac{2^{k}}{12} \rho,
\end{aligned}
$$

by using Proposition 3.4 with $r=2^{k} \rho$. 


\section{The packing lemma}

As explained in the introduction, if $\tau$ is the cover time of $\mathbb{D}$, then the set which is uncovered at time $\tau(1-\epsilon)$ consists (with high probability) of small and distant sets (this is Proposition 5.5). The proof of this is based on a second moment argument, which involves a sum over pairs of points in $\mathbb{D}$ (see Lemma 5.4). In order to get a good bound for this sum, we will have to get estimates on the maximal number of points in $\mathbb{D}$ within a certain distance of a fixed point $x \in \mathbb{D}$. In general, we need to obtain an estimate on the maximum number of $x \in \mathbb{D}$ that belongs to a certain bounded subset of $\mathbb{R}^{d}$. This is the main purpose of this section, and the result is presented in Lemma 4.2 below, sometimes referred to as the packing lemma. However, in order to prove this lemma it is also convenient to introduce the concept of a maximal (in the sense that it contains the maximum number of points), $\rho$-separated subset of a bounded set $A$. This subset will play a crucial role in transferring results for finite sets (i.e. Theorem 5.7) to the non-discrete case (i.e. Theorem 1.1). However, obtaining a suitable $\rho$-separated set is somewhat technical as we will now see.

Recall (from the Introduction) the definition of a $\rho$-separated set. Let $\mathcal{A}_{1}$ denote the set of measurable $A \subset \mathbb{R}^{d}$ such that the diameter $\operatorname{diam}(A)=1$ and the centre of mass of $A$ is the origin $o$. It is not straightforward to define a choice function which assigns a maximal $\rho$-separated subset to every $A \in \mathcal{A}_{1}$.

For fixed $A \in \mathcal{A}_{1}$ and $\rho>0$, we let $\mathcal{R}_{A, \rho}$ denote the collection of $\rho$-separated subsets of $A$ which contains the maximum number of points. Clearly, $\mathcal{R}_{A, \rho}$ is always non-empty, and in general, $\mathcal{R}_{A, \rho}$ is an uncountable collection of $\rho$-separated subsets of $A$. Then, we use the axiom of choice to pick a member $\mathbb{A}^{\rho}$ from every $\mathcal{R}_{A, \rho}$. From this choice, we define the "discretization" operator $\Delta$ which maps $(A, \rho) \in \mathcal{A}_{1} \times \mathbb{R}^{+}$ to the corresponding maximal $\rho$-separated set so that $\mathbb{A}^{\rho}=\Delta(A, \rho)$. Since we will often think of $\rho>0$ as being fixed, it will be convenient to write $\Delta^{\rho}(A)$ in place of $\Delta(A, \rho)$.

Our next step is to enlarge the domain of $\Delta^{\rho}$ from $\mathcal{A}_{1}$ to the collection of all bounded measurable subsets of $\mathbb{R}^{d}$. We will do this in a particular way to ensure a specific invariance property (i.e. (4.1)). For any bounded and measurable set $B$, and any $\alpha, y>0$, we let $\alpha B=\{\alpha x: x \in B\}$ and $B-y=\{x-y: x \in B\}$. We will

also let $\operatorname{cm}(B)$ denote the centre of mass of $B$, and we note that $\frac{B-\operatorname{cm}(B)}{\operatorname{diam}(B)} \in \mathcal{A}_{1}$. Then, we define

$$
\Delta^{\rho}(B):=\operatorname{diam}(B) \Delta^{\rho / \operatorname{diam}(B)}\left(\frac{B-\operatorname{cm}(B)}{\operatorname{diam}(B)}\right)+\operatorname{cm}(B),
$$

and we will often write $\mathbb{B}^{\rho}$ in place of $\Delta^{\rho}(B)$. The main point of defining $\Delta^{\rho}$ in this way, is that it is easily checked that for any $\alpha>0$, and any bounded $A \subset \mathbb{R}^{d}$,

$$
\alpha \mathbb{A}^{\rho}=\alpha \Delta^{\rho}(A)=\Delta^{\alpha \rho}(\alpha A) .
$$

The interpretation of (4.1) is simply that the discrete $\rho$-separated subset obtained from $A$ by $\Delta^{\rho}$, scaled by $\alpha>0$ (so that the separation is now $\alpha \rho$ ), is the same as if we started with the $(\alpha-)$ scaled version of $A$, and then took the discrete $\alpha \rho$-separated subset.

The introduction of $\Delta^{\rho}$ will have the added benefit of also facilitating what would otherwise be overly cumbersome notation; it is more convenient to write $\Delta^{\rho}(A \cup B)$ or $\Delta^{\rho}(\alpha A)$ than any alternative. 
Our first lemma establishes three results for $\mathbb{A}^{\rho}$. This lemma will be used repeatedly in Section 6, but it will also be used in the proof of Lemma 4.2.

\section{Lemma 4.1.}

a) For any $A \subset \mathbb{R}^{d}$ bounded and all $\rho<\delta$ we have that $\left|\mathbb{A}^{\delta}\right| \leq\left|\mathbb{A}^{\rho}\right|$.

b) Let $A_{1} \cap A_{2}=\emptyset$ be bounded sets. Then we have that $\left|\Delta^{\rho}\left(A_{1} \cup A_{2}\right)\right| \leq$ $\left|\mathbb{A}_{1}^{\rho}\right|+\left|\mathbb{A}_{2}^{\rho}\right|$.

c) For any $A \subset \mathbb{R}^{d}$ bounded we have that for every $0<\rho<1$,

$$
\left|\mathbb{A}^{\rho}\right| \leq 6^{d} \rho^{-d}\left|\mathbb{A}^{1}\right|
$$

Proof. For part $a$ ), simply observe that any $\delta$-separated set is also $\rho$-separated. Thus, $\left|\mathbb{A}^{\delta}\right| \leq \max \{|\mathbb{D}|: \mathbb{D} \subset A, \operatorname{Sep}(\mathbb{D}) \geq \rho\}=\left|\mathbb{A}^{\rho}\right|$.

For part $b)$, assume that $\left|\Delta^{\rho}\left(A_{1} \cup A_{2}\right)\right|>\left|\mathbb{A}_{1}^{\rho}\right|+\left|\mathbb{A}_{2}^{\rho}\right|$. Then we get that

$$
\left|\mathbb{A}_{1}^{\rho}\right|+\left|\mathbb{A}_{2}^{\rho}\right|<\left|\Delta^{\rho}\left(A_{1} \cup A_{2}\right) \cap A_{1}\right|+\left|\Delta^{\rho}\left(A_{1} \cup A_{2}\right) \cap A_{2}\right|,
$$

and so without loss of generality we may assume that $\left|\mathbb{A}_{1}^{\rho}\right|<\left|\Delta^{\rho}\left(A_{1} \cup A_{2}\right) \cap A_{1}\right|$. However, $\Delta^{\rho}\left(A_{1} \cup A_{2}\right) \cap A_{1}$ is a $\rho$-separated set in $A_{1}$, so this would contradict the maximality of $\mathbb{A}_{1}^{\rho}$.

For part $c$ ), note first that $A \subset \bigcup_{x \in \mathbb{A}^{1}} B(x, 1)$. Next, let $x_{1}, \ldots x_{\left|\mathbb{A}^{1}\right|}$ be an enumeration of the points in the set $\mathbb{A}^{1}$. Set $D_{1}=B\left(x_{1}, 1\right)$ and then iteratively, let $D_{j}=B\left(x_{j}, 1\right) \backslash \cup_{i=1}^{j-1} B\left(x_{i}, 1\right)$. Obviously, $D_{j} \subset B\left(x_{j}, 1\right), D_{i} \cap D_{j}=\emptyset$ for $i \neq j$ and $A \subset \cup_{i=1}^{\left|\mathbb{A}^{1}\right|} B\left(x_{i}, 1\right)=\cup_{i=1}^{\left|\mathbb{A}^{1}\right|} D_{i}$. By using part $\left.b\right)$ of this lemma we have that

$$
\left|\mathbb{A}^{\rho}\right| \leq\left|\Delta^{\rho}\left(\bigcup_{i=1}^{\left|\mathbb{A}^{1}\right|} D_{i}\right)\right| \leq \sum_{i=1}^{\left|\mathbb{A}^{1}\right|}\left|\mathbb{D}_{i}^{\rho}\right| \leq\left|\mathbb{A}^{1}\right|\left|\mathbb{D}_{1}^{\rho}\right| .
$$

Consistent with our previous notation, let $\mathbb{B}(x, r)^{\rho}$ denote the maximal $\rho$-separated subset of the ball $B(x, r)$. We proceed to bound $\left|\mathbb{D}_{1}^{\rho}\right|=\left|\mathbb{B}(o, 1)^{\rho}\right|$. To that end, note that

$$
\bigcup_{x \in \mathbb{B}(o, 1)^{\rho}} B(x, \rho / 3) \subset B(o, 1+\rho)
$$

and that $B\left(x_{i}, \rho / 3\right) \cap B\left(x_{j}, \rho / 3\right)=\emptyset$ for all distinct points $x_{i}, x_{j} \in \mathbb{B}(o, 1)^{\rho}$, since $d\left(x_{i}, x_{j}\right) \geq \rho$ by the definition of $\mathbb{B}(o, 1)^{\rho}$. Therefore (recall that $\lambda_{d}$ denotes $d$ dimensional Lebesgue measure),

$$
\left|\mathbb{B}(o, 1)^{\rho}\right| \lambda_{d}\left(B\left(x_{1}, \rho / 3\right)\right) \leq \lambda_{d}(B(o, 1+\rho))=\kappa_{d}(1+\rho)^{d} \leq \kappa_{d} 2^{d},
$$

where we used $\rho<1$ in the last inequality. Since $\lambda_{d}\left(B\left(x_{1}, \rho / 3\right)\right)=\kappa_{d}(\rho / 3)^{d}$, it follows that $\left|\mathbb{B}(o, 1)^{\rho}\right| \leq 6^{d} \rho^{-d}$. Inserting this into (4.2) yields the result.

We are now ready to state and prove our packing lemma. Recall that $c$ represents a constant, depending only on the dimension $d$, and that it may change from line to line.

Lemma 4.2. There exists a constant $c<\infty$ depending on $d$ only such that for any $\mathbb{D}$ with $\operatorname{Sep}(\mathbb{D}) \geq \rho$ we have that

a) For any $\rho<r,|\mathbb{D} \cap B(o, r)| \leq c \rho^{-d} r^{d}$.

b) For any $y \in \mathbb{D}$ and $\rho<1$ we have that $\sum_{x \in \mathbb{D} \backslash B(y, 1)} d(y, x)^{1-d} \leq c \rho^{-d}|\mathbb{D}|^{1 / d}$. 
Proof: a) Similar to the proof above, let $\left\{x_{1}, \ldots, x_{N}\right\}=\mathbb{D} \cap B(o, r)$ and observe that $B\left(x_{i}, \rho\right) \subset B(o, r+\rho)$ for all $i=1, \ldots, N$. For any $j \neq i$ we have that $B\left(x_{i}, \rho / 3\right) \cap B\left(x_{j}, \rho / 3\right)=\emptyset$, since $d\left(x_{i}, x_{j}\right) \geq \rho$. Therefore,

$$
|\mathbb{D} \cap B(o, r)| \lambda_{d}\left(B\left(x_{1}, \rho / 3\right)\right) \leq \lambda_{d}(B(o, r+\rho))=\kappa_{d}(r+\rho)^{d} \leq c r^{d}
$$

where we used $\rho<r$ in the last inequality. Since $\lambda_{d}\left(B\left(x_{1}, \rho / 3\right)\right)=\kappa_{d}(\rho / 3)^{d}$ we have that

$$
|\mathbb{D} \cap B(o, r)| \leq c \rho^{-d} r^{d}
$$

and the proof is complete.

b) Let $N(\rho, r, \mathbb{D})=|\mathbb{D} \cap(B(o, r+1) \backslash B(o, r))|$. Our first step will be to estimate $N(\rho, r, \mathbb{D})$. Therefore, let $\left\{x_{1}, \ldots, x_{N(\rho, r, \mathbb{D})}\right\}$ denote the points in $\mathbb{D} \cap$ $(B(o, r+1) \backslash B(o, r))$. For any $i=1, \ldots, N(\rho, r, \mathbb{D})$ we have that $B\left(x_{i}, \rho / 3\right) \subset$ $(B(o, r+2) \backslash B(o, r-1))$. Also, for any $j \neq i$ we have that $B\left(x_{i}, \rho / 3\right) \cap B\left(x_{j}, \rho / 3\right)$ $=\emptyset$, since $d\left(x_{i}, x_{j}\right) \geq \rho$ by the assumption on $\mathbb{D}$. Thus,

$$
\begin{aligned}
& N(\rho, r, \mathbb{D}) \lambda_{d}\left(B\left(x_{1}, \rho / 3\right)\right) \\
& \quad \leq \lambda_{d}(B(o, r+2) \backslash B(o, r-1))=\kappa_{d}\left((r+2)^{d}-(r-1)^{d}\right) \leq c r^{d-1} .
\end{aligned}
$$

Since $\lambda_{d}\left(B\left(x_{1}, \rho / 3\right)\right)=\kappa_{d}(\rho / 3)^{d}$ we conclude that

$$
N(\rho, r, \mathbb{D}) \leq c \rho^{-d} r^{d-1}
$$

We assume without loss of generality that $y=o$. Order the points $\left\{y_{1}, \ldots, y_{M}\right\}=$ $\mathbb{D} \backslash\{o\}$ such that $d\left(o, y_{i}\right) \leq d\left(o, y_{j}\right)$ for all $i<j$. Then, let $N_{k}=\left|\mathbb{B}(o, k)^{\rho}\right|$ and observe that for any $i>N_{k}$ we must have that $d\left(o, y_{i}\right)>k$. Then, note that by Lemma 4.1 part $b$ ), and (4.3), we get that for $k \geq 2$,

$$
\begin{aligned}
N_{k}- & N_{k-1}=\left|\mathbb{B}(o, k)^{\rho}\right|-\left|\mathbb{B}(o, k-1)^{\rho}\right| \\
& \leq\left|\Delta^{\rho}(B(o, k) \backslash B(o, k-1))\right| \leq c \rho^{-d} k^{d-1} \leq c 2^{d-1} \rho^{-d}(k-1)^{d-1} .
\end{aligned}
$$

We now define $K:=\max \left\{k: N_{k}<|\mathbb{D}|\right\}$ and consider the box $S_{K}=$ $[-K / \sqrt{d}, K / \sqrt{d}]^{d} \subset B(o, K)$. We then have that $\left|\mathbb{S}_{K}^{\rho}\right| \leq\left|B(o, K)^{\rho}\right|=N_{K}$. Consider then the set $\rho \mathbb{Z}^{d} \cap S_{K}$ where $\rho \mathbb{Z}^{d}$ is the $d$-dimensional hypercubic lattice whose vertices are at distance $\rho$. Trivially, $\rho \mathbb{Z}^{d} \cap S_{K}$ is a $\rho$-separated set in $S_{K}$ and so we have that $\left|\mathbb{S}_{K}^{\rho}\right| \geq\left|\rho \mathbb{Z}^{d} \cap S_{K}\right| \geq c \rho^{-d} K^{d}$, by the maximality of $\left|\mathbb{S}_{K}^{\rho}\right|$. Therefore,

$$
c \rho^{-d} K^{d} \leq\left|\mathbb{S}_{K}^{\rho}\right| \leq N_{K}<|\mathbb{D}|
$$

and therefore, $K \leq c \rho|\mathbb{D}|^{1 / d}$. 
By part $a), N_{1} \leq c \rho^{-d}$ and so by letting $N_{0}=0$ and using Equation (4.4) we get that,

$$
\begin{aligned}
& \sum_{x \in \mathbb{D} \backslash B(o, 1)} d(o, x)^{1-d} \leq \sum_{i=1}^{M} \max \left(d\left(o, y_{i}\right), 1\right)^{1-d} \\
& \quad=\sum_{k=1}^{K+1} \sum_{i=N_{k-1}+1}^{\min \left(N_{k}, M\right)} \max \left(d\left(o, y_{i}\right), 1\right)^{1-d} \\
& \leq N_{1}+\sum_{k=2}^{K+1}\left(N_{k}-N_{k-1}\right)(k-1)^{1-d} \\
& \leq N_{1}+\sum_{k=2}^{K+1} c \rho^{-d}(k-1)^{d-1}(k-1)^{1-d} \\
& \leq c \rho^{-d}+K c \rho^{-d} \leq c \rho^{-d}+c \rho^{1-d}|\mathbb{D}|^{1 / d} \leq c \rho^{-d}|\mathbb{D}|^{1 / d}
\end{aligned}
$$

where we use that $\max \left(d\left(o, x_{i}\right), 1\right) \geq 1$ for $1 \leq i \leq N_{1}$ and the observation above that $d\left(o, x_{i}\right) \geq k-1$ for $N_{k-1}+1 \leq i \leq N_{k}$.

\section{The fluctuation theorem and proof of Theorem 1.2}

The purpose of this section is to prove Theorem 1.2. This will be done by proving Theorem 5.7, which provides a bound for the fluctuation of $\mathbb{P}((\mathcal{T}(\mathbb{D})-\log |\mathbb{D}| \leq z)$ from the Gumbel distribution function. As mentioned in the introduction, the strategy is similar to that of Belius (2012).

In our first result we compare the cover time of a set with prescribed separation, and the cover time of an "independent" (or infinitely separated if you will) set of the same cardinality. The proof uses Lemmas 3.2 and 3.3.

Proposition 5.1. Let $m>0$ and let $\mathbb{D}$ be a finite set in $\mathbb{R}^{d}$ such that $\operatorname{Sep}(\mathbb{D}) \geq$ $\max \left((n / 2)^{\frac{m+2}{d-1}}, 4\right)$. Then, for $t \geq 0$ we have that

$$
\left|\mathbb{P}(\mathcal{T}(\mathbb{D}) \leq t)-\mathbb{P}(\mathcal{T}(o) \leq t)^{n}\right| \leq t c(n / 2)^{-m} .
$$

Proof. Let $\mathbb{D}=\left\{x_{i}\right\}_{i=1}^{n}, \mathbb{D}_{i}:=\mathbb{D} \backslash\left\{x_{k}\right\}_{k=1}^{i}$ and $B_{i}=\bigcup_{x \in \mathbb{D}_{i}} B(x, 1)$. Then

$$
\begin{aligned}
& \left|\mathbb{P}(\mathcal{T}(\mathbb{D}) \leq t)-\mathbb{P}(\mathcal{T}(o) \leq t)^{n}\right| \\
& \quad \leq\left|\mathbb{P}(\mathcal{T}(\mathbb{D}) \leq t)-\mathbb{P}\left(\mathcal{T}\left(\mathbb{D}_{1}\right) \leq t\right) \mathbb{P}\left(\mathcal{T}\left(x_{1}\right) \leq t\right)\right| \\
& \quad+\mathbb{P}(\mathcal{T}(o) \leq t)\left|\mathbb{P}\left(\mathcal{T}\left(\mathbb{D}_{1}\right) \leq t\right)-\mathbb{P}(\mathcal{T}(o) \leq t)^{n-1}\right|,
\end{aligned}
$$

by translation invariance. Using Lemma 3.2, we get

$$
\left|\mathbb{P}(\mathcal{T}(\mathbb{D}) \leq t)-\mathbb{P}\left(\mathcal{T}\left(\mathbb{D}_{1}\right) \leq t\right) \mathbb{P}\left(\mathcal{T}\left(x_{1}\right) \leq t\right)\right| \leq 4 \mathbb{P}\left(\omega_{t}\left(\mathcal{L}_{B_{1}, B\left(x_{1}, 1\right)}\right) \neq 0\right),
$$

and thus

$$
\begin{aligned}
& \left|\mathbb{P}(\mathcal{T}(\mathbb{D}) \leq t)-\mathbb{P}(\mathcal{T}(o) \leq t)^{n}\right| \\
& \quad \leq 4 \mathbb{P}\left(\omega_{t}\left(\mathcal{L}_{B_{1}, B\left(x_{1}, 1\right)}\right) \neq 0\right)+\left|\mathbb{P}\left(\mathcal{T}\left(\mathbb{D}_{1}\right) \leq t\right)-\mathbb{P}(\mathcal{T}(o) \leq t)^{n-1}\right| .
\end{aligned}
$$

Repeating the same steps $n-1$ more times, we get:

$$
\begin{aligned}
& \left|\mathbb{P}(\mathcal{T}(\mathbb{D}) \leq t)-\mathbb{P}(\mathcal{T}(o) \leq t)^{n}\right| \\
& \quad \leq 4\left(\mathbb{P}\left(\omega_{t}\left(\mathcal{L}_{B_{1}, B\left(x_{1}, 1\right)}\right) \neq 0\right)+\cdots+\mathbb{P}\left(\omega_{t}\left(\mathcal{L}_{B\left(x_{n}, 1\right), B\left(x_{n-1}, 1\right)}\right) \neq 0\right)\right) .
\end{aligned}
$$


We let $r:=\operatorname{Sep}(\mathbb{D})$ (we chose not to use $\rho$, since here we think of the separation as being large rather than small). Applying Lemma 3.3 (which requires $r \geq 4$ ) to the above gives

$$
\begin{aligned}
& \left|\mathbb{P}(\mathcal{T}(\mathbb{D}) \leq t)-\mathbb{P}(\mathcal{T}(o) \leq t)^{n}\right| \\
& \quad \leq c\left((n-1) \frac{t}{r^{d-1}}+(n-2) \frac{t}{r^{d-1}}+\cdots+\frac{t}{r^{d-1}}\right) \\
& \quad=c \frac{n(n-1)}{2} \frac{t}{r^{d-1}} .
\end{aligned}
$$

Therefore,

$$
\left|\mathbb{P}(\mathcal{T}(\mathbb{D}) \leq t)-\mathbb{P}(\mathcal{T}(o) \leq t)^{n}\right| \leq n^{2} c \frac{t}{r^{d-1}} .
$$

Since $r \geq(n / 2)^{\frac{m+2}{d-1}}$, we have that $r^{-(d-1)} \leq(n / 2)^{-(m+2)}$ and thus

$$
\left|\mathbb{P}(\mathcal{T}(\mathbb{D}) \leq t)-\mathbb{P}(\mathcal{T}(o) \leq t)^{n}\right| \leq n^{2} c \frac{t}{r^{d-1}} \leq t c(n / 2)^{-m}
$$

Before we can proceed, we need to introduce some convenient notation. For $0<\epsilon<1$, let

$$
\mathbb{D}_{\epsilon}:=\{x \in \mathbb{D}: \mathcal{T}(x)>(1-\epsilon) \log |\mathbb{D}|\}
$$

for any finite set $\mathbb{D}$. The set $\mathbb{D}_{\epsilon}$ corresponds to the subset of points $x \in \mathbb{D}$ that are not covered at time $(1-\epsilon) \log |\mathbb{D}|$. Furthermore, we will let

$$
C_{d}:=\sqrt{1-4^{-1 /(d-2)}}
$$

where we interpret $C_{2}=1$. Furthermore, let

$$
\tilde{C}_{d}:=\frac{C_{d}}{12\left(1+C_{d}\right)} .
$$

Our next aim is Lemma 5.4, which will provide bounds on two sums (over pairs of points in $\mathbb{D}$ ) of the probabilities of two distinct points being in the set $\mathbb{D}_{\epsilon}$. This lemma is a preparation for the second moment argument of Proposition 5.5 (mentioned in the introduction). The strategy of the proof of Lemma 5.4 is to split the sum into three parts. The first part is a sum over all pairs of points that are within distance $C_{d}$ of each other, the second sums over pairs within the intermediate region between $C_{d}$ and $\log |\mathbb{D}|$, while the last sums over distant points. In order to obtain the desired result, we then use properties of $\mu_{d-1}$ along with the packing Lemma 4.2 and the estimate provided in Proposition 3.4.

As we have mentioned before, our key results require that our sets are separated "enough". To that end we shall have need of the following definition.

Definition 5.2. Let $\mathbb{D}$ be a finite set and let $\rho=\operatorname{Sep}(\mathbb{D})$. We say that $\mathbb{D}$ is good if it satisfies the following conditions: $|\mathbb{D}|^{\rho / 800} \geq 2,|\mathbb{D}|^{1 /(2 d)} \geq 4$ and $\rho^{-d}(\log |\mathbb{D}|)^{d} \leq$ $|\mathbb{D}|^{\tilde{C}_{d} / 2}$.

Remarks: The definition of $\mathbb{D}$ being good might look somewhat artificial. However, it will be convenient to have such a definition as we will appeal to it on many occasions below. It can be useful to simply think of a good set $\mathbb{D}$ as being "large enough" and "separated enough", and the notion of goodness makes this precise. 
The following lemma is stated without a proof as it is trivial to check. One might argue that the condition in this lemma can be taken as the definition of good. However, it will be more convenient to have the three explicit conditions of Definition 5.2 at hand.

Lemma 5.3. There exists a constant $D=D(d)<\infty$ such that any $\mathbb{D}$ with

$$
\operatorname{Sep}(\mathbb{D}) \geq \log 2 \frac{800}{\log |\mathbb{D}|}
$$

and $|\mathbb{D}| \geq D$ is good.

Lemma 5.4. Let $\mathbb{D}$ be a good set. Then, for every $\epsilon$ such that $\epsilon<\min \left(\frac{\rho}{36}, \frac{5}{36}, \frac{C_{d}}{148}\right)$ and $|\mathbb{D}|^{\epsilon} \geq 2$ we have that
a) $\sum_{\substack{x, y \in \mathbb{D} \\ x \neq y}} \mathbb{P}\left(x, y \in \mathbb{D}_{\epsilon}\right)<c|\mathbb{D}|^{-\epsilon}+|\mathbb{D}|^{2 \epsilon}$
b) $\sum_{\substack{x, y \in \mathbb{D} \\ 0<d(x, y)<b|\mathbb{D}|^{1 / 2 d}}} \mathbb{P}\left(x, y \in \mathbb{D}_{\epsilon}\right)<c b^{d}|\mathbb{D}|^{-\epsilon}$,

for every $b \geq 1$, and where $c$ is a constant depending only on the dimension $d$.

Remark: If it is the case that $\rho$ is so small that no such $\epsilon$ exists, then the statement is vacuous. A similar comment applies to other statements below.

Proof. We will prove both statements by considering the sum

$$
I=\sum_{\substack{x, y \in \mathbb{D} \\ 0<d(x, y) \leq a}} \mathbb{P}\left(x, y \in \mathbb{D}_{\epsilon}\right)
$$

and choosing appropriate values for $a$ later. Let us split $I$ into three parts:

$$
\begin{gathered}
I_{1}=\sum_{\substack{x, y \in \mathbb{D} \\
\rho \leq d(x, y)<C_{d}}} \mathbb{P}\left(x, y \in \mathbb{D}_{\epsilon}\right), \\
I_{2}=\sum_{\substack{x, y \in \mathbb{D} \\
C_{d} \leq d(x, y)<\log |\mathbb{D}|}} \mathbb{P}\left(x, y \in \mathbb{D}_{\epsilon}\right),
\end{gathered}
$$

and

$$
I_{3}=\sum_{\substack{x, y \in \mathbb{D} \\ \log |\mathbb{D}| \leq d(x, y) \leq a}} \mathbb{P}\left(x, y \in \mathbb{D}_{\epsilon}\right) .
$$

Of course, it could be that $\rho \geq C_{d}$ or even that $\rho \geq \log |\mathbb{D}|$. If so, $I_{1}$ and/or $I_{2}$ are simply zero while the analysis of $I_{3}$ remains the same. We will therefore assume that $\rho<C_{d}$ without any further comment.

In all three sums, we will use the following.

$$
\begin{aligned}
\mathbb{P}(x, y & \left.\in \mathbb{D}_{\epsilon}\right) \\
& =\mathbb{P}(\{\mathcal{T}(x)>(1-\epsilon) \log |\mathbb{D}|\} \cap\{\mathcal{T}(y)>(1-\epsilon) \log |\mathbb{D}|\}) \\
& =\mathbb{P}\left(\omega_{(1-\epsilon) \log |\mathbb{D}|}\left(\mathcal{L}_{B(x, 1)} \cup \mathcal{L}_{B(y, 1)}\right)=0\right) \\
& =\exp \left(-(1-\epsilon) \log |\mathbb{D}| \mu_{d, 1}\left(\mathcal{L}_{B(x, 1)} \cup \mathcal{L}_{B(y, 1)}\right)\right) .
\end{aligned}
$$

The sum $I_{1}$ : Let $K=\left\lfloor\frac{\log C_{d}-\log \rho}{\log 2}\right\rfloor$, where $\lfloor\cdot\rfloor$ denotes the integer part. Note that

$$
2^{K+1} \rho \leq 2^{\frac{\log C_{d}-\log \rho}{\log 2}+1} \rho=2 C_{d} \text { while } 2^{K+1} \rho \geq 2^{\frac{\log C_{d}-\log \rho}{\log 2}} \rho=C_{d} .
$$


Note also that $K \geq 0$ since $\rho<C_{d}$. Thus, (5.4) becomes

$$
I_{1}=\sum_{\substack{x, y \in \mathbb{D} \\ \rho \leq d(x, y)<C_{d}}} \mathbb{P}\left(x, y \in \mathbb{D}_{\epsilon}\right) \leq \sum_{k=0}^{K} \sum_{\substack{x, y \in \mathbb{D} \\ 2^{k} \rho \leq d(x, y)<2^{k+1} \rho}} \mathbb{P}\left(x, y \in \mathbb{D}_{\epsilon}\right) .
$$

For $2^{k} \rho \leq d(x, y)<2^{k+1} \rho$, we have that

$$
\begin{aligned}
\mu_{d, 1} & \left(\mathcal{L}_{B(x, 1)} \cup \mathcal{L}_{B(y, 1)}\right)=\mu_{d, 1}\left(\mathcal{L}_{B(o, 1)} \cup \mathcal{L}_{B\left(d(x, y) e_{1}, 1\right)}\right) \\
& =\mu_{d, 1}\left(\mathcal{L}_{B(o, 1)}\right)+\mu_{d, 1}\left(\mathcal{L}_{B\left(d(x, y) e_{1}, 1\right)}\right)-\mu_{d, 1}\left(\mathcal{L}_{B(o, 1)} \cap \mathcal{L}_{B\left(d(x, y) e_{1}, 1\right)}\right) \\
& \geq \mu_{d, 1}\left(\mathcal{L}_{B(o, 1)}\right)+\mu_{d, 1}\left(\mathcal{L}_{B\left(2^{k} \rho e_{1}, 1\right)}\right)-\mu_{d, 1}\left(\mathcal{L}_{B(o, 1)} \cap \mathcal{L}_{B\left(2^{k} \rho e_{1}, 1\right)}\right) \\
& =\mu_{d, 1}\left(\mathcal{L}_{B(o, 1)} \cup \mathcal{L}_{B\left(2^{k} \rho e_{1}, 1\right)}\right)
\end{aligned}
$$

where the inequality follows since any line $L \in \mathcal{L}_{B(o, 1)} \cap \mathcal{L}_{B\left(d(x, y) e_{1}, 1\right)}$ must also belong to $\mathcal{L}_{B(o, 1)} \cap \mathcal{L}_{B\left(2^{k} \rho e_{1}, 1\right)}$. Thus

$$
\begin{aligned}
\exp & \left(-(1-\epsilon) \log |\mathbb{D}| \mu_{d, 1}\left(\mathcal{L}_{B(x, 1)} \cup \mathcal{L}_{B(y, 1)}\right)\right) \\
& \leq \exp \left(-(1-\epsilon) \log |\mathbb{D}| \mu_{d, 1}\left(\mathcal{L}_{B(o, 1)} \cup \mathcal{L}_{B\left(2^{k} \rho e_{1}, 1\right)}\right)\right) \\
& =\exp (-(1-\epsilon) \log |\mathbb{D}| \beta(\rho, k)) .
\end{aligned}
$$

Therefore, (5.7), (5.8) and the above gives us that

$$
\begin{aligned}
I_{1} \leq & \sum_{k=0}^{K} \sum_{\substack{x, y \in \mathbb{D} \\
2^{k} \rho \leq d(x, y)<2^{k+1} \rho}} \exp (-(1-\epsilon) \log |\mathbb{D}| \beta(\rho, k)) \\
& \leq \sum_{k=0}^{K}|\mathbb{D}|^{1-(1-\epsilon)\left(1+\frac{2^{k}}{12} \rho\right)} c\left(2^{k+1} \rho\right)^{d} \rho^{-d},
\end{aligned}
$$

where we used Lemma 4.2 part $a$ ) and Lemma 3.5 (which we can use since $2^{K} \rho \leq C_{d}$ as noted above). Furthermore, for any $k$ we claim that

$$
1-(1-\epsilon)\left(1+\frac{2^{k}}{12} \rho\right)=-\frac{2^{k}}{12} \rho+\epsilon+\frac{2^{k}}{12} \epsilon \rho \leq-2^{k} \epsilon .
$$

Indeed, (5.9) is equivalent to

$$
\epsilon \leq \rho \frac{\frac{1}{12} 2^{k}}{1+\frac{1}{12} 2^{k} \rho+2^{k}}=\rho \frac{\frac{1}{12}}{2^{-k}+\frac{1}{12} \rho+1},
$$

and this holds since $\epsilon \leq \frac{\rho}{36} \leq \rho \frac{\frac{1}{12}}{2^{-k}+\frac{1}{12} \rho+1}$ by our assumption on $\epsilon$. Using (5.9) we get that

$$
\begin{aligned}
I_{1} \leq & c \sum_{k=0}^{K}|\mathbb{D}|^{-2^{k} \epsilon} 2^{d(k+1)} \leq c|\mathbb{D}|^{-\epsilon} \sum_{k=0}^{\infty}|\mathbb{D}|^{-\left(2^{k}-1\right) \epsilon} 2^{d(k+1)} \\
& =c|\mathbb{D}|^{-\epsilon}\left(2^{d}+|\mathbb{D}|^{-\epsilon} 2^{2 d}+\sum_{k=2}^{\infty}|\mathbb{D}|^{-\left(2^{k}-1\right) \epsilon} 2^{d(k+1)}\right) \\
& \leq c|\mathbb{D}|^{-\epsilon}\left(1+\sum_{k=1}^{\infty}|\mathbb{D}|^{-2^{k} \epsilon} 2^{d k}\right) .
\end{aligned}
$$


Furthermore,

$$
\begin{aligned}
& \sum_{k=1}^{\infty}|\mathbb{D}|^{-2^{k} \epsilon} 2^{d k} \leq c \sum_{k=1}^{\infty} \int_{2^{k-1}}^{2^{k}}|\mathbb{D}|^{-x \epsilon} x^{d-1} \mathrm{~d} x \leq c \int_{0}^{\infty} e^{-x \epsilon \log |\mathbb{D}|} x^{d-1} \mathrm{~d} x \\
& =c\left[x^{d-1} \frac{e^{-x \epsilon \log |\mathbb{D}|}}{-\epsilon \log |\mathbb{D}|}\right]_{0}^{\infty}+c \frac{d-1}{\epsilon \log |\mathbb{D}|} \int_{0}^{\infty} e^{-x \epsilon \log |\mathbb{D}|} x^{d-2} \mathrm{~d} x \\
& =\cdots=\frac{c}{\left(\log |\mathbb{D}|^{\epsilon}\right)^{d}},
\end{aligned}
$$

and by assumption $\log |\mathbb{D}|^{\epsilon} \geq \log 2$ and so

$$
I_{1} \leq c|\mathbb{D}|^{-\epsilon} .
$$

The sum $I_{2}$ : Consider now Equation (5.5). Equation (5.7) implies that

$$
\begin{aligned}
I_{2}= & \sum_{\substack{x, y \in \mathbb{D} \\
C_{d} \leq d(x, y)<\log |\mathbb{D}|}} \mathbb{P}\left(x, y \in \mathbb{D}_{\epsilon}\right) \\
= & \sum_{\substack{x, y \in \mathbb{D} \\
C_{d} \leq d(x, y)<\log |\mathbb{D}|}} \exp \left(-(1-\epsilon) \log |\mathbb{D}| \mu_{d, 1}\left(\mathcal{L}_{B(o, 1)} \cup \mathcal{L}_{B\left(d(x, y) e_{1}, 1\right)}\right)\right) \\
\leq & \sum_{\substack{x, y \in \mathbb{D} \\
C_{d} \leq d(x, y)<\log |\mathbb{D}|}} \exp \left(-(1-\epsilon) \log |\mathbb{D}| \mu_{d, 1}\left(\mathcal{L}_{B(o, 1)} \cup \mathcal{L}_{B\left(C_{d} e_{1}, 1\right)}\right)\right),
\end{aligned}
$$

since $d(x, y) \geq C_{d}$. Now, Lemma 3.5 applied to $\rho=C_{d}$ and $k=0$, implies that $\mu_{d, 1}\left(\mathcal{L}_{B(o, 1)} \cup \mathcal{L}_{B\left(C_{d} e_{1}, 1\right)}\right) \geq 1+\frac{C_{d}}{12}$, which gives

$$
\begin{aligned}
I_{2} \leq & \sum_{\substack{x, y \in \mathbb{D} \\
C_{d} \leq d(x, y)<\log |\mathbb{D}|}} \exp \left(-(1-\epsilon) \log |\mathbb{D}| \mu_{d, 1}\left(\mathcal{L}_{B(o, 1)} \cup \mathcal{L}_{B\left(C_{d} e_{1}, 1\right)}\right)\right) \\
\leq & c|\mathbb{D}| \rho^{-d}(\log |\mathbb{D}|)^{d} \exp \left(-(1-\epsilon)\left(1+C_{d} / 12\right) \log |\mathbb{D}|\right) \\
& =c \rho^{-d}(\log |\mathbb{D}|)^{d}|\mathbb{D}|^{\epsilon-C_{d} / 12+\epsilon C_{d} / 12} \leq c|\mathbb{D}|^{\epsilon-\tilde{C}_{d} / 2+\epsilon \tilde{C}_{d}}
\end{aligned}
$$

where we used Lemma 4.2 part $a$ ) in the second inequality and the goodness of $\mathbb{D}$ (in order to estimate $\rho^{-d}(\log |\mathbb{D}|)^{d}$ ) in the last inequality. Furthermore, we claim that $\epsilon-\tilde{C}_{d} / 2+\epsilon \tilde{C}_{d} \leq-\epsilon$. Indeed, this follows since

$$
\frac{\tilde{C}_{d}}{2\left(2+\tilde{C}_{d}\right)}=\frac{C_{d}}{2\left(24\left(1+C_{d}\right)+C_{d}\right)} \geq \frac{C_{d}}{148} \geq \epsilon
$$

by our assumption on $\epsilon$. We conclude that

$$
I_{2} \leq c|\mathbb{D}|^{-\epsilon} .
$$

The sum $I_{3}$ : We now turn to Equation (5.6) and the sum $I_{3}$. Here we have that $d(x, y) \geq \log |\mathbb{D}| \geq \log 4^{2 d} \geq 4$ by the goodness of $\mathbb{D}$. Therefore, we can apply Lemma 3.1 to see that

$$
\begin{aligned}
& \mu_{d, 1}\left(\mathcal{L}_{B(x, 1)} \cup \mathcal{L}_{B(y, 1)}\right) \\
& \quad=2 \mu_{d, 1}\left(\mathcal{L}_{B(x, 1)}\right)-\mu_{d, 1}\left(\mathcal{L}_{B(x, 1)} \cap \mathcal{L}_{B(y, 1)}\right) \geq 2-\frac{c_{2}}{d(x, y)^{d-1}} .
\end{aligned}
$$


Then, we see from (5.6) that

$$
\begin{aligned}
I_{3} \leq & \sum_{\substack{x, y \in \mathbb{D} \\
\log |\mathbb{D}| \leq d(x, y) \leq a}} \exp \left(-(1-\epsilon) \log |\mathbb{D}|\left(2-\frac{c_{2}}{d(x, y)^{d-1}}\right)\right) \\
& =|\mathbb{D}|^{-2(1-\epsilon)} \sum_{\substack{x, y \in \mathbb{D} \\
\log |\mathbb{D}| \leq d(x, y) \leq a}} \exp \left((1-\epsilon) \log |\mathbb{D}| c_{2} d(x, y)^{1-d}\right) .
\end{aligned}
$$

Since $\log |\mathbb{D}| \leq d(x, y)$, we have that

$$
(1-\epsilon) \log |\mathbb{D}| c_{2} d(x, y)^{1-d} \leq(1-\epsilon) c_{2}(\log |\mathbb{D}|)^{2-d} \leq c_{2} .
$$

It is easy to prove that for any $x \leq c_{2}$ we must have that $e^{x} \leq 1+e^{c_{2}} x$, and so we get that

$$
\begin{aligned}
& |\mathbb{D}|^{-2(1-\epsilon)} \sum_{\substack{x, y \in \mathbb{D} \\
\log |\mathbb{D}| \leq d(x, y) \leq a}} \exp \left((1-\epsilon) \log |\mathbb{D}| c_{2} d(x, y)^{1-d}\right) \\
& \leq|\mathbb{D}|^{-2(1-\epsilon)} \sum_{\substack{x, y \in \mathbb{D} \\
\log |\mathbb{D}| \leq d(x, y) \leq a}}\left(1+e^{c_{2}}(1-\epsilon) \log |\mathbb{D}| d(x, y)^{1-d}\right) \\
& \leq \min \left(|\mathbb{D}|^{2 \epsilon}, c \rho^{-d}|\mathbb{D}|^{2 \epsilon-1} a^{d}\right) \\
& +c(1-\epsilon)|\mathbb{D}|^{-2(1-\epsilon)} \log |\mathbb{D}| \sum_{\substack{x, y \in \mathbb{D} \\
\log |\mathbb{D}| \leq d(x, y) \leq a}} d(x, y)^{1-d}
\end{aligned}
$$

where the minimum comes from summing 1 and using part $a$ ) of Lemma 4.2 to bound the number of elements in the sum.

Next, we provide a bound to the last term on the right hand side of (5.12). To that end, note that

$$
\sum_{\substack{x, y \in \mathbb{D} \\ \log |\mathbb{D}| \leq d(x, y) \leq a}} d(x, y)^{1-d} \leq \sum_{x \in \mathbb{D}} \sum_{y \in \mathbb{D} \backslash B(x, 1)} d(x, y)^{1-d} \leq \sum_{x \in \mathbb{D}} c \frac{|\mathbb{D}|^{1 / d}}{\rho^{d}} \leq c \frac{|\mathbb{D}|^{1+1 / d}}{\rho^{d}},
$$

where we used part $b$ ) of Lemma 4.2 in the second inequality. Furthermore, this and the fact that $\rho^{-d}(\log |\mathbb{D}|)^{d} \leq|\mathbb{D}|^{\tilde{C}_{d} / 2}$ by the goodness of $\mathbb{D}$ implies that

$$
\begin{aligned}
c(1-\epsilon)|\mathbb{D}|^{-2(1-\epsilon)} \log |\mathbb{D}| \sum_{\substack{x, y \in \mathbb{D} \\
\log |\mathbb{D}| \leq d(x, y) \leq a}} d(x, y)^{1-d} \\
\leq c(1-\epsilon) \rho^{-d} \log |\mathbb{D}||\mathbb{D}|^{-2(1-\epsilon)+1+1 / d} \\
\leq c \rho^{-d}(\log |\mathbb{D}|)^{d}|\mathbb{D}|^{-2(1-\epsilon)+1+1 / d} \\
\leq c|\mathbb{D}|^{-2(1-\epsilon)+1+1 / d+\tilde{C}_{d} / 2} \leq c|\mathbb{D}|^{-\epsilon} .
\end{aligned}
$$

where we in the last inequality use that $-2(1-\epsilon)+1+1 / d+\tilde{C}_{d} / 2 \leq-\epsilon$. Indeed, this follows since

$$
1-\frac{1}{d}-\frac{\tilde{C}_{d}}{2}=1-\frac{1}{d}-\frac{C_{d}}{24\left(1+C_{d}\right)} \geq 1-\frac{1}{d}-\frac{2}{24} \geq \frac{5}{12} \geq 3 \epsilon,
$$


by our assumption on $\epsilon$. Combining (5.13) and (5.12) we see that

$$
I_{3}<\min \left(|\mathbb{D}|^{2 \epsilon}, c \rho^{-d}|\mathbb{D}|^{2 \epsilon-1} a^{d}\right)+c|\mathbb{D}|^{-\epsilon} .
$$

By summing the contributions from $I_{1}, I_{2}$ and $I_{3}((5.10),(5.11)$ and $(5.14))$, we then conclude that

$$
I=\sum_{\substack{x, y \in \mathbb{D} \\ 0<d(x, y) \leq a}} \mathbb{P}\left(x, y \in \mathbb{D}_{\epsilon}\right)<\min \left(|\mathbb{D}|^{2 \epsilon}, c \rho^{-d}|\mathbb{D}|^{2 \epsilon-1} a^{d}\right)+c|\mathbb{D}|^{-\epsilon} .
$$

Taking the limit $a \rightarrow \infty$, we obtain

$$
\sum_{\substack{x, y \in \mathbb{D} \\ x \neq y}} \mathbb{P}\left(x, y \in \mathbb{D}_{\epsilon}\right)<|\mathbb{D}|^{2 \epsilon}+c|\mathbb{D}|^{-\epsilon}
$$

and the first statement is proved.

Furthermore, taking $a=b|\mathbb{D}|^{1 / 2 d}$, we have that

$$
\begin{gathered}
c \rho^{-d}|\mathbb{D}|^{2 \epsilon-1} a^{d}=c \rho^{-d}|\mathbb{D}|^{2 \epsilon-1 / 2} b^{d}=c b^{d}|\mathbb{D}|^{-\epsilon}\left(\rho^{-d}|\mathbb{D}|^{3 \epsilon-1 / 2}\right) \\
\leq c b^{d}|\mathbb{D}|^{-\epsilon}\left(|\mathbb{D}|^{3 \epsilon-1 / 2+\tilde{C}_{d} / 2}\right) \leq c b^{d}|\mathbb{D}|^{-\epsilon},
\end{gathered}
$$

where we used that $\rho^{-d} \leq \rho^{-d}(\log |\mathbb{D}|)^{d} \leq|\mathbb{D}|^{\tilde{C}_{d} / 2}$ by the goodness of $\mathbb{D}$ and that $|\mathbb{D}|^{3 \epsilon-1 / 2+\tilde{C}_{d} / 2} \leq 1$ (which as above follows by our assumption that $\epsilon<5 / 36$ ). Thus

$$
\sum_{\substack{x, y \in \mathbb{D} \\(x, y)<b|\mathbb{D}|^{1 / 2 d}}} \mathbb{P}\left(x, y \in \mathbb{D}_{\epsilon}\right)<c b^{d}|\mathbb{D}|^{-\epsilon}
$$

and the proof is complete.

The next proposition is another crucial step towards the proof of Theorem 1.2. Consider first

$$
\begin{aligned}
& G_{\mathbb{D}, \epsilon}=\left\{K \subset \mathbb{D}:\left.\left.|| K|-| \mathbb{D}\right|^{\epsilon}|\leq| \mathbb{D}\right|^{2 \epsilon / 3}\right. \text { and } \\
& \left.\quad d(x, y) \geq|\mathbb{D}|^{1 / 2 d} \text { for all distinct } x, y \in K\right\},
\end{aligned}
$$

so that $G_{\mathbb{D}, \epsilon}$ is a collection of subsets of $\mathbb{D}$ that are well separated and close in cardinality to $|\mathbb{D}|^{\epsilon}$. Also note that the condition $\left.\left.|| K|-| \mathbb{D}\right|^{\epsilon}|\leq| \mathbb{D}\right|^{2 \epsilon / 3}$ implies that $G_{\mathbb{D}, \epsilon}$ consists only of non-empty sets.

Proposition 5.5. Let $\mathbb{D}$ be a good set. Then, for every positive $\epsilon$ such that $\epsilon<$ $\min \left(\frac{\rho}{36}, \frac{5}{36}, \frac{C_{d}}{148}\right)$ and $|\mathbb{D}|^{\epsilon} \geq 2$ we have that

$$
\mathbb{P}\left(\mathbb{D}_{\epsilon} \notin G_{\mathbb{D}, \epsilon}\right) \leq c|\mathbb{D}|^{-\epsilon / 3} .
$$

Here, the constant c only depends on $d$.

Proof: We will get the result by proving that

$$
\mathbb{P}\left(\exists x, y \in \mathbb{D}_{\epsilon}: 0<d(x, y)<|\mathbb{D}|^{1 / 2 d}\right) \leq c|\mathbb{D}|^{-\epsilon},
$$

and

$$
\mathbb{P}\left(\left.\left.|| \mathbb{D}_{\epsilon}|-| \mathbb{D}\right|^{\epsilon}|>| \mathbb{D}\right|^{2 \epsilon / 3}\right) \leq c|\mathbb{D}|^{-\epsilon / 3}
$$


For (5.16) we have:

$$
\begin{aligned}
\mathbb{P}\left(\exists x, y \in \mathbb{D}_{\epsilon}: 0<d(x, y)<|\mathbb{D}|^{1 / 2 d}\right) & \\
= & \mathbb{P}\left(\bigcup_{\substack{x, y \in \mathbb{D} \\
0<d(x, y)<|\mathbb{D}|^{1 / 2 d}}}\left\{x, y \in \mathbb{D}_{\epsilon}\right\}\right) \\
\leq & \sum_{\substack{x, y \in \mathbb{D} \\
0<d(x, y)<|\mathbb{D}|^{1 / 2 d}}} \mathbb{P}\left(x, y \in \mathbb{D}_{\epsilon}\right) \leq c|\mathbb{D}|^{-\epsilon},
\end{aligned}
$$

where the last inequality come from part $b$ ) of Lemma 5.4 with $b=1$, and (5.16) is done.

For (5.17), we observe that since $\mathbb{P}(\mathcal{T}(x)>t)=e^{-t}$,

$$
\mathbb{E}\left(\left|\mathbb{D}_{\epsilon}\right|\right)=\sum_{x \in \mathbb{D}} \mathbb{P}(\mathcal{T}(x)>(1-\epsilon) \log |\mathbb{D}|)=|\mathbb{D}| \exp (-(1-\epsilon) \log |\mathbb{D}|)=|\mathbb{D}|^{\epsilon}
$$

By Chebyshev's inequality,

$$
\mathbb{P}\left(\left.\left.|| \mathbb{D}_{\epsilon}|-| \mathbb{D}\right|^{\epsilon}|>| \mathbb{D}\right|^{2 \epsilon / 3}\right) \leq \frac{\mathbb{E}\left|\mathbb{D}_{\epsilon}\right|^{2}-|\mathbb{D}|^{2 \epsilon}}{|\mathbb{D}|^{4 \epsilon / 3}}
$$

Now

$$
\begin{aligned}
\mathbb{E}\left|\mathbb{D}_{\epsilon}\right|^{2} & =\sum_{x, y \in \mathbb{D}} \mathbb{P}\left(x, y \in \mathbb{D}_{\epsilon}\right) \\
& =\sum_{x \in \mathbb{D}} \mathbb{P}\left(x \in \mathbb{D}_{\epsilon}\right)+\sum_{\substack{x, y \in \mathbb{D} \\
x \neq y}} \mathbb{P}\left(x, y \in \mathbb{D}_{\epsilon}\right) \leq|\mathbb{D}|^{\epsilon}+c|\mathbb{D}|^{-\epsilon}+|\mathbb{D}|^{2 \epsilon},
\end{aligned}
$$

by part $a$ ) of Lemma 5.4. Plugging this into (5.18) yields

$$
\begin{aligned}
& \mathbb{P}\left(\left.\left.|| \mathbb{D}_{\epsilon}|-| \mathbb{D}\right|^{\epsilon}|>| \mathbb{D}\right|^{2 \epsilon / 3}\right) \\
& \quad \leq \frac{|\mathbb{D}|^{\epsilon}+c|\mathbb{D}|^{-\epsilon}+|\mathbb{D}|^{2 \epsilon}-|\mathbb{D}|^{2 \epsilon}}{|\mathbb{D}|^{4 \epsilon / 3}} \leq|\mathbb{D}|^{-\epsilon / 3}+c|\mathbb{D}|^{-7 \epsilon / 3} \leq c|\mathbb{D}|^{-\epsilon / 3}
\end{aligned}
$$

and the proof is complete.

We shall make use of the following inequality which we state here (without argument) for convenience.

$$
-x-x^{2} \leq \log (1-x) \leq-x \text { for every } x \in[0,1 / 2]
$$

We need a simple auxiliary result before we can present our fluctuation theorem.

Lemma 5.6. Let $\mathbb{D}$ be a finite set, and let $\epsilon>0$ be such that $|\mathbb{D}|^{\epsilon} \geq 4$. We then have that for any $z \geq-\frac{\epsilon}{4} \log |\mathbb{D}|$

$$
\left|\left(1-\frac{e^{-z}}{|\mathbb{D}|^{\epsilon}}\right)^{|\mathbb{D}|^{\epsilon}+|\mathbb{D}|^{2 \epsilon / 3}}-\exp \left(-e^{-z}\right)\right| \leq 3|\mathbb{D}|^{-\epsilon / 12},
$$


and similarly,

$$
\left|\left(1-\frac{e^{-z}}{|\mathbb{D}|^{\epsilon}}\right)^{|\mathbb{D}|^{\epsilon}-|\mathbb{D}|^{2 \epsilon / 3}}-\exp \left(-e^{-z}\right)\right| \leq|\mathbb{D}|^{-\epsilon / 12} .
$$

Remark: As the proof of this lemma is a straightforward, albeit somewhat tedious, exercise we shall only provide a sketch.

Sketch of proof. We only address (5.20), as (5.21) is proved in essentially the same way.

Let $a=e^{-z}$ and $b=|\mathbb{D}|^{\epsilon}$, and note that $a / b \leq|\mathbb{D}|^{-3 \epsilon / 4} \leq 1 / 2$ by the assumption on $z$ and since $|\mathbb{D}|^{\epsilon} \geq 4$. Using this and (5.19), it is then elementary to prove that

$$
\left(1-\frac{a}{b}\right)^{b+b^{2 / 3}} \leq \exp (-a)
$$

One can then continue to show that

$$
\begin{aligned}
& \left|\exp (-a)-\left(1-\frac{a}{b}\right)^{b+b^{2 / 3}}\right| \\
& \quad \leq \exp (-a)\left(1-\exp \left(-\left(\frac{a^{2}}{b}+\frac{a}{b^{1 / 3}}+\frac{a^{2}}{b^{4 / 3}}\right)\right)\right) \leq\left(1-\exp \left(-3 b^{-1 / 12}\right)\right),
\end{aligned}
$$

where we use (5.22), the lower bound in (5.19) and the definitions of $a, b$. Finally, it is easy to see that

$$
\left(1-\exp \left(-3 b^{-1 / 12}\right)\right) \leq 3 b^{-1 / 12} \leq 3|\mathbb{D}|^{-\epsilon / 12} .
$$

Thus, (5.20) is proved.

We are now ready to prove a theorem which gives an explicit bound on the difference between the (centred) cover time $\mathcal{T}(\mathbb{D})$ of a good set $\mathbb{D}$, and the distribution function of a Gumbel distribution.

Theorem 5.7. For any good set $\mathbb{D}$ with $\rho=\operatorname{Sep}(\mathbb{D})$ and any $\epsilon$ such that $|\mathbb{D}|^{\epsilon} \geq 2^{16}$ and $\epsilon<\min \left(\frac{\rho}{36}, \frac{1}{12}, \frac{C_{d}}{148}\right)$ we have that

$$
\sup _{z \in \mathbb{R}}\left|\mathbb{P}(\mathcal{T}(\mathbb{D})-\log |\mathbb{D}| \leq z)-\exp \left(-e^{-z}\right)\right| \leq c_{3}|\mathbb{D}|^{-\epsilon / 12},
$$

where $c_{3}$ is a constant depending on d only.

Remarks: Note that the Theorem is only useful if $|\mathbb{D}|^{-\epsilon / 12}$ is small or goes to 0 for a sequence $\left(\mathbb{D}_{n}\right)_{n \geq 1}$. Thus, the assumption that $|\mathbb{D}|^{\epsilon} \geq 2^{16}$ (which can be somewhat relaxed with additional work) will turn out not to be a limitation.

Proof: We are going to split the proof into three cases and start with the easy ones. Case 1: Consider $z \leq-\frac{\epsilon}{4} \log |\mathbb{D}|$. Then

$$
\begin{aligned}
& \mathbb{P}(\mathcal{T}(\mathbb{D}) \leq(\log |\mathbb{D}|+z)) \leq \mathbb{P}\left(\mathcal{T}(\mathbb{D}) \leq\left(\log |\mathbb{D}|-\frac{\epsilon}{4} \log |\mathbb{D}|\right)\right) \\
& \quad=\mathbb{P}\left(\left\{x \in \mathbb{D}: \mathcal{T}(x)>\left(1-\frac{\epsilon}{4}\right) \log |\mathbb{D}|\right\}=\emptyset\right)=\mathbb{P}\left(\mathbb{D}_{\epsilon / 4}=\emptyset\right) .
\end{aligned}
$$

We have that $\mathbb{P}\left(\mathbb{D}_{\epsilon / 4}=\emptyset\right) \leq \mathbb{P}\left(\mathbb{D}_{\epsilon / 4} \notin G_{\mathbb{D}, \epsilon / 4}\right)$, since $G_{\mathbb{D}, \epsilon / 4}$ only contains nonempty sets. Thus, by Proposition 5.5 we have that,

$$
\mathbb{P}(\mathcal{T}(\mathbb{D}) \leq(\log |\mathbb{D}|+z)) \leq \mathbb{P}\left(\mathbb{D}_{\epsilon / 4} \notin G_{\mathbb{D}, \epsilon / 4}\right) \leq c|\mathbb{D}|^{-\epsilon / 12}
$$


Using that $z \leq-\log |\mathbb{D}|^{\epsilon / 4}$ we have that $\exp \left(-e^{-z}\right) \leq \exp \left(-|\mathbb{D}|^{\epsilon / 4}\right) \leq|\mathbb{D}|^{-\epsilon / 4}$. Therefore,

$$
\begin{aligned}
& \left|\mathbb{P}(\mathcal{T}(\mathbb{D}) \leq(\log |\mathbb{D}|+z))-\exp \left(-e^{-z}\right)\right| \\
& \quad \leq \mathbb{P}(\mathcal{T}(\mathbb{D}) \leq(\log |\mathbb{D}|+z))+\exp \left(-e^{-z}\right) \\
& \quad \leq c|\mathbb{D}|^{-\epsilon / 12}+|\mathbb{D}|^{-\epsilon / 4}<c|\mathbb{D}|^{-\epsilon / 12}
\end{aligned}
$$

and this ends the first case.

Case 2: Assume $z \geq \epsilon \log |\mathbb{D}|$. This gives

$$
\begin{aligned}
& \mathbb{P}(\mathcal{T}(\mathbb{D})>(\log |\mathbb{D}|+z)) \leq \mathbb{P}(\mathcal{T}(\mathbb{D})>(1+\epsilon) \log |\mathbb{D}|) \\
& \quad=\mathbb{P}\left(\bigcup_{x \in \mathbb{D}}\{\mathcal{T}(x)>(1+\epsilon) \log |\mathbb{D}|\}\right) \leq|\mathbb{D}| \mathbb{P}(\mathcal{T}(o)>(1+\epsilon) \log |\mathbb{D}|) \\
& \quad=|\mathbb{D}| \exp (-(1+\epsilon) \log |\mathbb{D}|)=|\mathbb{D}|^{-\epsilon} .
\end{aligned}
$$

Using that $z \geq \epsilon \log |\mathbb{D}|$ we have that $\exp \left(-e^{-z}\right) \geq \exp \left(-|\mathbb{D}|^{-\epsilon}\right) \geq 1-|\mathbb{D}|^{-\epsilon}$ by the inequality $e^{x} \geq 1+x$, which holds for all $x$. This, and the above equation gives

$$
\begin{aligned}
& \left|\mathbb{P}(\mathcal{T}(\mathbb{D}) \leq(\log |\mathbb{D}|+z))-\exp \left(-e^{-z}\right)\right| \\
& \quad=\left|1-\mathbb{P}(\mathcal{T}(\mathbb{D})>(\log |\mathbb{D}|+z))-\exp \left(-e^{-z}\right)\right| \\
& \quad \leq \mathbb{P}(\mathcal{T}(\mathbb{D})>(\log |\mathbb{D}|+z))+\left|1-\exp \left(-e^{-z}\right)\right| \leq|\mathbb{D}|^{-\epsilon}+|\mathbb{D}|^{-\epsilon},
\end{aligned}
$$

which proves the case $z \geq \epsilon \log |\mathbb{D}|$.

Case 3: Assume that $z \in\left(-\frac{\epsilon}{4} \log |\mathbb{D}|, \epsilon \log |\mathbb{D}|\right)$ and start by observing that

$$
\begin{aligned}
\left|\mathbb{P}(\mathcal{T}(\mathbb{D}) \leq \log |\mathbb{D}|+z)-\exp \left(-e^{-z}\right)\right| \\
\leq\left|\mathbb{P}(\mathcal{T}(\mathbb{D}) \leq \log |\mathbb{D}|+z)-\mathbb{P}\left(\mathcal{T}(\mathbb{D}) \leq \log |\mathbb{D}|+z, \mathbb{D}_{\epsilon} \in G_{\mathbb{D}, \epsilon}\right)\right| \\
\quad+\left|\exp \left(-e^{-z}\right) \mathbb{P}\left(\mathbb{D}_{\epsilon} \in G_{\mathbb{D}, \epsilon}\right)-\exp \left(-e^{-z}\right)\right| \\
\quad+\left|\mathbb{P}\left(\mathcal{T}(\mathbb{D}) \leq \log |\mathbb{D}|+z, \mathbb{D}_{\epsilon} \in G_{\mathbb{D}, \epsilon}\right)-\exp \left(-e^{-z}\right) \mathbb{P}\left(\mathbb{D}_{\epsilon} \in G_{\mathbb{D}, \epsilon}\right)\right| .
\end{aligned}
$$

We will now consider the three terms on the right hand side.

To deal with the first term, note simply that

$$
\begin{aligned}
& \left|\mathbb{P}(\mathcal{T}(\mathbb{D}) \leq \log |\mathbb{D}|+z)-\mathbb{P}\left(\mathcal{T}(\mathbb{D}) \leq \log |\mathbb{D}|+z, \mathbb{D}_{\epsilon} \in G_{\mathbb{D}, \epsilon}\right)\right| \\
& \quad=\mathbb{P}\left(\mathcal{T}(\mathbb{D}) \leq \log |\mathbb{D}|+z, \mathbb{D}_{\epsilon} \notin G_{\mathbb{D}, \epsilon}\right) \leq \mathbb{P}\left(\mathbb{D}_{\epsilon} \notin G_{\mathbb{D}, \epsilon}\right) \leq c|\mathbb{D}|^{-\epsilon / 3}
\end{aligned}
$$

by Proposition 5.5 .

For the second term of the right hand side in (5.26), note that

$$
\left|\exp \left(-e^{-z}\right) \mathbb{P}\left(\mathbb{D}_{\epsilon} \in G_{\mathbb{D}, \epsilon}\right)-\exp \left(-e^{-z}\right)\right|=\exp \left(-e^{-z}\right) \mathbb{P}\left(\mathbb{D}_{\epsilon} \notin G_{\mathbb{D}, \epsilon}\right) \leq c|\mathbb{D}|^{-\epsilon / 3}
$$

again by Proposition 5.5.

We now turn to the third term of the right hand side in (5.26), and this is where all our previous efforts come together. We will show that for any $\mathbb{K} \in G_{\mathbb{D}, \epsilon}$,

$$
\left|\mathbb{P}\left(\mathcal{T}(\mathbb{D}) \leq \log |\mathbb{D}|+z \mid \mathbb{D}_{\epsilon}=\mathbb{K}\right)-\exp \left(-e^{-z}\right)\right| \leq c|\mathbb{D}|^{-\epsilon / 12} .
$$

Then, multiplying by $\mathbb{P}\left(\mathbb{D}_{\epsilon}=\mathbb{K}\right)$ and summing over all $\mathbb{K} \in G_{\mathbb{D}, \epsilon}$ on both sides, we get

$$
\left|\mathbb{P}\left(\mathcal{T}(\mathbb{D}) \leq \log |\mathbb{D}|+z, \mathbb{D}_{\epsilon} \in G_{\mathbb{D}, \epsilon}\right)-\exp \left(-e^{-z}\right) \mathbb{P}\left(\mathbb{D}_{\epsilon} \in G_{\mathbb{D}, \epsilon}\right)\right| \leq c|\mathbb{D}|^{-\epsilon / 12} .
$$


We can then conclude from (5.26), that

$$
\left|\mathbb{P}(\mathcal{T}(\mathbb{D}) \leq(\log |\mathbb{D}|+z))-\exp \left(-e^{-z}\right)\right| \leq c|\mathbb{D}|^{-\epsilon / 12}
$$

for all $z \in\left(-\frac{\epsilon}{4} \log |\mathbb{D}|, \epsilon \log |\mathbb{D}|\right)$, and the proof will be complete.

In order to prove (5.27), define

$$
\omega^{1}=\left\{L \in \omega_{(1-\epsilon) \log |\mathbb{D}|}: \exists x \in \mathbb{D} \text { such that } x \in \mathfrak{c}(L)\right\},
$$

so that $\omega^{1}$ is the subset of $\omega_{(1-\epsilon)} \log |\mathbb{D}|$ consisting of the cylinders actually covering a point $x \in \mathbb{D}$. Similarly, let

$$
\omega^{2}=\left\{L \in \omega_{(1-\epsilon) \log |\mathbb{D}|, \log |\mathbb{D}|+z}: \exists x \in \mathbb{D} \text { such that } x \in \mathfrak{c}(L)\right\}
$$

so that a cylinder $\mathfrak{c}(L) \in \omega^{2}$ arrives between times $(1-\epsilon) \log |\mathbb{D}|$ and $(\log |\mathbb{D}|+z)$, and in addition covers some $x \in \mathbb{D}$. Finally, let $\omega^{3}=\omega^{1} \cup \omega^{2}$. For any $\omega \in \Omega$, let $\mathcal{C}(\omega, \mathbb{D})$ be the set of points in $\mathbb{D}$ that are covered by the cylinders in $\omega$.

Fix $\mathbb{K} \in G_{\mathbb{D}, \epsilon}$ and define the event

$$
E_{1}:=\left\{\mathbb{D}_{\epsilon}=\mathbb{K}\right\}=\left\{\mathbb{D} \backslash \mathbb{K}=\mathcal{C}\left(\omega^{1}, \mathbb{D}\right)\right\} .
$$

The equality holds since $\mathbb{K} \subset \mathbb{D}$ is the uncovered set iff $\mathbb{D} \backslash \mathbb{K}$ is the covered set. Furthermore, $\{\mathcal{T}(\mathbb{D}) \leq \log |\mathbb{D}|+z\}$ is the event that all the points of $\mathbb{D}$ are covered by time $(\log |\mathbb{D}|+z)$. Hence, by the definition of $\omega^{3},\{\mathcal{T}(\mathbb{D}) \leq \log |\mathbb{D}|+z\}=\{\mathbb{D}=$ $\left.\mathcal{C}\left(\omega^{3}, \mathbb{D}\right)\right\}$ so that $E_{1} \cap\{\mathcal{T}(\mathbb{D}) \leq \log |\mathbb{D}|+z\}=E_{1} \cap\left\{\mathbb{K} \subset \mathcal{C}\left(\omega^{2}, \mathbb{D}\right)\right\}$. Letting $E_{2}=\left\{\mathbb{K} \subset \mathcal{C}\left(\omega^{2}, \mathbb{D}\right)\right\}$ and using that $\omega^{1}$ and $\omega^{2}$ are independent, we have that $\mathbb{P}\left(E_{1} \cap E_{2}\right)=\mathbb{P}\left(E_{1}\right) \mathbb{P}\left(E_{2}\right)$. Furthermore, since $\omega^{2}$ has the same distribution as a Poisson line process with intensity $\epsilon \log |\mathbb{D}|+z$, we get that

$$
\mathbb{P}\left(E_{2}\right)=\mathbb{P}\left(\mathbb{K} \subset \mathcal{C}\left(\omega^{2}, \mathbb{D}\right)\right)=\mathbb{P}(\mathcal{T}(\mathbb{K}) \leq \epsilon \log |\mathbb{D}|+z) .
$$

On the other hand,

$$
\begin{aligned}
\mathbb{P}\left(E_{2}\right) & =\frac{\mathbb{P}\left(E_{1} \cap E_{2}\right)}{\mathbb{P}\left(E_{1}\right)} \\
= & \frac{\mathbb{P}\left(E_{1} \cap\{\mathcal{T}(\mathbb{D}) \leq \log |\mathbb{D}|+z\}\right)}{\mathbb{P}\left(E_{1}\right)}=\mathbb{P}\left(\mathcal{T}(\mathbb{D}) \leq \log |\mathbb{D}|+z \mid E_{1}\right),
\end{aligned}
$$

so we conclude that

$$
\mathbb{P}\left(\mathcal{T}(\mathbb{D}) \leq \log |\mathbb{D}|+z \mid \mathbb{D}_{\epsilon}=\mathbb{K}\right)=\mathbb{P}(\mathcal{T}(\mathbb{K}) \leq \epsilon \log |\mathbb{D}|+z)
$$

Therefore, using (5.29) we have

$$
\begin{aligned}
\left|\mathbb{P}\left(\mathcal{T}(\mathbb{D}) \leq \epsilon \log |\mathbb{D}|+z \mid \mathbb{D}_{\epsilon}=\mathbb{K}\right)-\exp \left(-e^{-z}\right)\right| \\
=\left|\mathbb{P}(\mathcal{T}(\mathbb{K}) \leq \epsilon \log |\mathbb{D}|+z)-\exp \left(-e^{-z}\right)\right| \\
\leq\left|\mathbb{P}(\mathcal{T}(\mathbb{K}) \leq \epsilon \log |\mathbb{D}|+z)-\mathbb{P}(\mathcal{T}(o) \leq \epsilon \log |\mathbb{D}|+z)^{|\mathbb{K}|}\right| \\
\quad+\left|\mathbb{P}(\mathcal{T}(o) \leq \epsilon \log |\mathbb{D}|+z)^{|\mathbb{K}|}-\exp \left(-e^{-z}\right)\right| .
\end{aligned}
$$

We will deal with the two terms on the right hand side of (5.30) separately.

For the first term, let $x, y \in \mathbb{K}$ be distinct. By the definition of $G_{\mathbb{D}, \epsilon}$ we have $d(x, y) \geq|\mathbb{D}|^{1 /(2 d)}=\left(|\mathbb{D}|^{\epsilon}\right)^{1 /(2 \epsilon d)}$. Furthermore, since $\left.\left.|| \mathbb{K}|-| \mathbb{D}\right|^{\epsilon}|\leq| \mathbb{D}\right|^{2 \epsilon / 3}$, we have that

$$
|\mathbb{D}|^{\epsilon}-|\mathbb{D}|^{2 \epsilon / 3} \leq|\mathbb{K}| \leq|\mathbb{D}|^{\epsilon}+|\mathbb{D}|^{2 \epsilon / 3} .
$$


In particular, Equation (5.31) implies $|\mathbb{K}| \leq 2|\mathbb{D}|^{\epsilon}$, so that $d(x, y) \geq\left(|\mathbb{D}|^{\epsilon}\right)^{1 /(2 \epsilon d)} \geq$ $(|\mathbb{K}| / 2)^{1 /(2 \epsilon d)}$. If we let $m=\frac{1}{2 \epsilon} \frac{d-1}{d}-2$, we conclude that $d(x, y) \geq(|\mathbb{K}| / 2)^{\frac{2+m}{d-1}}$. Moreover, by the goodness of $\mathbb{D}$ we have that $d(x, y) \geq|\mathbb{D}|^{1 /(2 d)} \geq 4$ and so we can use Proposition 5.1 with $n=|\mathbb{K}|$ together with the fact that $z \leq \epsilon \log |\mathbb{D}|$ to get

$$
\left|\mathbb{P}(\mathcal{T}(\mathbb{K}) \leq \epsilon \log |\mathbb{D}|+z)-\mathbb{P}(\mathcal{T}(o) \leq \epsilon \log |\mathbb{D}|+z)^{|\mathbb{K}|}\right| \leq c \epsilon \log |\mathbb{D}|(|\mathbb{K}| / 2)^{-m}
$$

Furthermore, since $|\mathbb{D}|^{\epsilon / 3} \geq 2^{16 / 3} \geq 2$ by assumption, we see that

$$
\frac{2}{|\mathbb{K}|} \leq \frac{2}{|\mathbb{D}|^{\epsilon}-|\mathbb{D}|^{2 \epsilon / 3}} \leq \frac{1}{|\mathbb{D}|^{\epsilon / 3}}
$$

where we use (5.31) in the first inequality and the fact that $x^{3}-x^{2} \geq 2 x$ if $x \geq 2$ in the second. Furthermore, since also $\epsilon<\frac{1}{12} \leq \frac{d-1}{6 d}$ by assumption, we get that $m>1$. We conclude that $(|\mathbb{K}| / 2)^{-m} \leq|\mathbb{D}|^{-m \epsilon / 3} \leq|\mathbb{D}|^{-\epsilon / 3}$. Thus,

$$
c \epsilon \log |\mathbb{D}|(|\mathbb{K}| / 2)^{-m} \leq c \epsilon(\log |\mathbb{D}|)|\mathbb{D}|^{-\epsilon / 3} .
$$

Using that $\log x \leq x^{1 / 4}$ for any $x \geq 2^{16}$ and using the assumption that $|\mathbb{D}|^{\epsilon} \geq$ $2^{16}$, we see that $\epsilon \log |\mathbb{D}| \leq|\mathbb{D}|^{\epsilon / 4}$ so that $c \epsilon(\log |\mathbb{D}|)|\mathbb{D}|^{-\epsilon / 3} \leq c|\mathbb{D}|^{\epsilon / 4}|\mathbb{D}|^{-\epsilon / 3}=$ $c|\mathbb{D}|^{-\epsilon / 12}$. We conclude from (5.32) and (5.33) that

$$
\left|\mathbb{P}(\mathcal{T}(\mathbb{K}) \leq \epsilon \log |\mathbb{D}|+z)-\mathbb{P}(\mathcal{T}(o) \leq \epsilon \log |\mathbb{D}|+z)^{|\mathbb{K}|}\right| \leq c|\mathbb{D}|^{-\epsilon / 12} .
$$

We can now turn to the second term of (5.30). We have that

$$
\mathbb{P}(\mathcal{T}(o) \leq \epsilon \log |\mathbb{D}|+z)^{|\mathbb{K}|}=(1-\exp (-\epsilon \log |\mathbb{D}|-z))^{|\mathbb{K}|}=\left(1-\frac{e^{-z}}{|\mathbb{D}|^{\epsilon}}\right)^{|\mathbb{K}|} .
$$

Then by (5.31),

$$
\left(1-\frac{e^{-z}}{|\mathbb{D}|^{\epsilon}}\right)^{|\mathbb{D}|^{\epsilon}+|\mathbb{D}|^{2 \epsilon / 3}} \leq \mathbb{P}(\mathcal{T}(o) \leq \epsilon \log |\mathbb{D}|+z)^{|\mathbb{K}|} \leq\left(1-\frac{e^{-z}}{|\mathbb{D}|^{\epsilon}}\right)^{|\mathbb{D}|^{\epsilon}-|\mathbb{D}|^{2 \epsilon / 3}} .
$$

Then, since $|\mathbb{D}|^{\epsilon} \geq 2^{16} \geq 4$ and since we are assuming that $z \geq-\frac{\epsilon}{4} \log |\mathbb{D}|$, we can use Lemma 5.6 to get that (with the obvious meaning of \pm )

$$
\begin{aligned}
& \left|\mathbb{P}(\mathcal{T}(o) \leq \epsilon \log |\mathbb{D}|+z)^{|\mathbb{K}|}-\exp \left(-e^{-z}\right)\right| \\
& \quad \leq\left|\left(1-\frac{e^{-z}}{|\mathbb{D}|^{\epsilon}}\right)^{|\mathbb{D}|^{\epsilon} \pm|\mathbb{D}|^{2 \epsilon / 3}}-\exp \left(-e^{-z}\right)\right| \leq c|\mathbb{D}|^{-\epsilon / 12} .
\end{aligned}
$$

Combining this with (5.30), (5.34) and (5.35), the inequality (5.27) is proved. This completes the proof.

The proof of Theorem 1.2 is now easy.

Proof of Theorem 1.2. Let $\rho_{n}=\operatorname{Sep}\left(\mathbb{D}_{n}\right)$ and let

$$
\epsilon_{n}:=\min \left(\frac{\rho_{n}}{50}, \frac{1}{24}, \frac{C_{d}}{150}\right) .
$$

Note that

$$
\lim _{n \rightarrow \infty}\left|\mathbb{D}_{n}\right|^{\rho_{n}}=\infty
$$


by assumption. We want to apply Theorem 5.7 (for every $n$ large enough), and therefore we need to verify the three conditions of that theorem.

Firstly, by using (5.36) together with Lemma 5.3 we conclude that there exists some $N<\infty$ such that $\mathbb{D}_{n}$ is good for every $n \geq N$.

Secondly, we clearly have that

$$
\epsilon_{n}<\min \left(\frac{\rho_{n}}{36}, \frac{1}{12}, \frac{C_{d}}{148}\right) .
$$

Thirdly, we can again use (5.36) together with the definition of $\epsilon_{n}$ to see that $\lim _{n \rightarrow \infty}\left|\mathbb{D}_{n}\right|^{\epsilon_{n}}=\infty$. Thus, by perhaps increasing $N$ even further we have that $\left|\mathbb{D}_{n}\right|^{\epsilon_{n}} \geq 2^{16}$ for every $n \geq N$.

We can therefore apply Theorem 5.7 to conclude that

$$
\sup _{z \in \mathbb{R}}\left|\mathbb{P}\left(\mathcal{T}\left(\mathbb{D}_{n}\right)-\log \left|\mathbb{D}_{n}\right| \leq z\right)-\exp \left(-e^{-z}\right)\right| \leq c_{3}\left|\mathbb{D}_{n}\right|^{-\epsilon_{n} / 12},
$$

for every $n \geq N$. The statement now follows since $\lim _{n \rightarrow \infty}\left|\mathbb{D}_{n}\right|^{\epsilon_{n}}=\infty$.

\section{Proof of Theorem 1.1}

In order to prove Theorem 1.1, we will study two quantities that are closely related to $\mathcal{T}(A)$. Firstly, recall the definition of $\mathbb{A}^{\rho}$ from Section 4 . We will think of $\mathcal{T}(A)$ and $\mathcal{T}\left(\mathbb{A}^{\rho}\right)$ as being generated by the same cylinder process, and sometimes we will write $\mathcal{T}\left(\mathbb{A}^{\rho},\left(\omega_{t}\right)_{t \geq 0}\right)$ for emphasis. We have the following easy proposition which we will not use, but we include for completeness and motivation.

Proposition 6.1. We have that for any $\rho>0$,

$$
\mathcal{T}\left(\mathbb{A}^{\rho}\right) \leq \limsup _{\delta \rightarrow 0} \mathcal{T}\left(\mathbb{A}^{\delta}\right)=\mathcal{T}(A),
$$

where the inequality and equality holds for a.e. $\left(\omega_{t}\right)_{t \geq 0}$.

Proof. Obviously, $\mathcal{T}\left(\mathbb{A}^{\rho},\left(\omega_{t}\right)_{t \geq 0}\right) \leq \mathcal{T}\left(A,\left(\omega_{t}\right)_{t \geq 0}\right)$ for every $\rho>0$. Assume that $\mathcal{T}\left(A,(\omega)_{t \geq 0}\right)>\tau$ and let

$$
\mathcal{C}_{\tau}:=\bigcup_{(L, s) \in \Psi: s \leq \tau} \mathfrak{c}(L)
$$

Then, there exists a point $x \in A \backslash \mathcal{C}_{\tau}$ and some $\delta>0$ such that $B(x, \delta) \subset \mathbb{R}^{d} \backslash \mathcal{C}_{\tau}$. This follows since a.s. $\mathcal{C}_{\tau}$ is a closed set. Therefore we must have that $\mathcal{T}\left(\mathbb{A}^{\delta / 2}\right)>\tau$. We conclude that $\lim \sup _{\delta \rightarrow 0} \mathcal{T}\left(\mathbb{A}^{\delta}\right) \geq \mathcal{T}(A)$.

Of course, Proposition 6.1 tells us that in order to get a good approximation of $\mathcal{T}(A)$ from below, one should estimate by $\mathcal{T}\left(\mathbb{A}^{\rho}\right)$ and take $\rho$ as small as possible. However, by taking $\rho$ too small, the estimates that we will obtain for $\mathcal{T}\left(\mathbb{A}^{\rho}\right)$ from Theorem 5.7 will become useless. Therefore, it will be important to pick $\rho$ in an optimal way. Later, we shall see that the "sweet-spot" is provided by letting $\rho$ be of order $\left(\log \left|\mathbb{A}^{1}\right|\right)^{-1}$.

In order to define our second quantity, we start by saying that a ball $B(x, \rho)$ is singularly covered by time $t$ if there exists $L \in \omega_{t}$ such that $B(x, \rho) \subset \mathfrak{c}(L)$. 
Alternatively, the point $x$ is $\rho$-singularly covered by time $t$ if the corresponding ball $B(x, \rho)$ is singularly covered. Thus,

$$
\mathcal{T}_{s}^{\rho}(x):=\inf \left\{t>0: \exists L \in \omega_{t} \text { such that } B(x, \rho) \subset \mathfrak{c}(L)\right\}
$$

is then the time at which the point $x$ is $\rho$-singularly covered. Finally, we define the $(\rho-)$ well cover time

$$
\mathcal{T}_{w}^{\rho}(A):=\inf \left\{t>0: \mathcal{T}_{s}^{\rho}(x) \leq t \forall x \in \mathbb{A}^{\rho}\right\} .
$$

Again, $\mathcal{T}_{w}^{\rho}(A)$ is generated by using the same cylinder process used to generate $\mathcal{T}(A)$ and $\mathcal{T}\left(\mathbb{A}^{\rho}\right)$. It is an easy consequence of the above definitions that

$$
\mathcal{T}\left(\mathbb{A}^{\rho},\left(\omega_{t}\right)_{t \geq 0}\right) \leq \mathcal{T}\left(A,\left(\omega_{t}\right)_{t \geq 0}\right) \leq \mathcal{T}_{w}^{\rho}\left(A,\left(\omega_{t}\right)_{t \geq 0}\right),
$$

and these inequalities will provide the bridge between the fluctuation result for discrete sets, i.e. Theorem 5.7 and Theorem 1.1. For the lower bound this is obvious, while for the upper we shall have use of the following proposition.

Proposition 6.2. We have that

$$
(1-\rho)^{d-1} \mathcal{T}_{w}^{\rho}(A) \stackrel{d}{=} \mathcal{T}\left(\Delta^{\rho /(1-\rho)}\left((1-\rho)^{-1} A\right)\right),
$$

where $\stackrel{d}{=}$ denotes equality in distribution.

Proof. Firstly, observe that the $\rho$-well cover time of $A$ equals the cover time of $\mathbb{A}^{\rho}$, provided that the cylinders in our cylinder process were of radius $1-\rho$ instead of 1. Let $\mathcal{T}^{1-\rho}\left(\mathbb{A}^{\rho}\right)$ denote this cover time so that

$$
\mathcal{T}^{1-\rho}\left(\mathbb{A}^{\rho}\right)=\mathcal{T}_{w}^{\rho}(A)
$$

Secondly, we can scale space by a factor of $(1-\rho)^{-1}$ in order to re-obtain a cylinder process of radius 1 . However, this scaling results in a cylinder process of rate $(1-\rho)^{d-1}$ (rather than 1 ). Indeed, start by recalling the notation from Section 2.1 and the meaning of $\Delta^{\rho}$ from Section 4. As in the proof of Proposition 3.4, observe that for any fixed $L \in G(d, 1)$ the set of $y \in L^{\perp}$ such that $L+y \in \mathcal{L}_{K}$ is $\Pi_{L^{\perp}}(K)$. Furthermore, we see that

$$
\int_{L^{\perp}} \mathbb{1}\left(L+y \in \mathcal{L}_{K}\right) \lambda_{d-1}(\mathrm{~d} y)=\lambda_{d-1}\left(\Pi_{L^{\perp}}(K)\right)
$$

for any compact $K \subset \mathbb{R}^{d}$. Thus, by equation (2.1),

$$
\begin{aligned}
\mu_{d, 1} & \left(\mathcal{L}_{K /(1-\rho)}\right)=\frac{1}{\kappa_{d-1}} \int_{G(d, 1)} \lambda_{d-1}\left(\Pi_{L^{\perp}}(K /(1-\rho))\right) \nu_{d, 1}(\mathrm{~d} L) \\
= & \frac{1}{\kappa_{d-1}(1-\rho)^{d-1}} \int_{G(d, 1)} \lambda_{d-1}\left(\Pi_{L^{\perp}}(K)\right) \nu_{d, 1}(\mathrm{~d} L)=\frac{\mu_{d, 1}\left(\mathcal{L}_{K}\right)}{(1-\rho)^{d-1}},
\end{aligned}
$$

from which the scaling claim follows. Of course, after this scaling is performed, the set $\mathbb{A}^{\rho}$ is now $\rho /(1-\rho)$-separated and we obtain that (see (4.1))

$$
(1-\rho)^{-1} \mathbb{A}^{\rho}=\Delta^{\rho /(1-\rho)}\left((1-\rho)^{-1} A\right) .
$$

Therefore, the cover time $\mathcal{T}^{1-\rho}\left(\mathbb{A}^{\rho}\right)$ equals the cover time of $\Delta^{\rho /(1-\rho)}\left((1-\rho)^{-1} A\right)$ when using a Poisson cylinder process with radius 1 and intensity $(1-\rho)^{d-1}$. Thus,

$$
(1-\rho)^{d-1} \mathcal{T}_{w}^{\rho}(A)=(1-\rho)^{d-1} \mathcal{T}^{1-\rho}\left(\mathbb{A}^{\rho}\right) \stackrel{d}{=} \mathcal{T}\left(\Delta^{\rho /(1-\rho)}\left((1-\rho)^{-1} A\right)\right),
$$

as desired. 
Remark: Intuitively, when $\rho$ is small there will be little difference between $\rho$ and $\rho /(1-\rho)$ and so

$$
(1-\rho)^{-(d-1)} \mathcal{T}\left(\Delta^{\rho /(1-\rho)}\left((1-\rho)^{-1} A\right)\right) \approx \mathcal{T}\left(\mathbb{A}^{\rho}\right) .
$$

This means that we should be able to "almost" match the upper and lower bound in (6.2). Of course, this is indeed the strategy of the proof of Theorem 1.1.

Before we proceed, we observe that for any $A$ and $\rho>0$,

$$
N_{2 \rho}(A) \leq\left|\mathbb{A}^{\rho}\right| \leq N_{\rho /(2 \sqrt{d})}(A) .
$$

Indeed, a ball of radius $\rho$ can be inscribed within a box of side-length $2 \rho$, and this gives the lower bound. Furthermore, a box of side-length $\frac{\rho}{2 \sqrt{d}}$ has diameter $\rho / 2$, and therefore it cannot contain more than one point of $\mathbb{A}^{\rho}$. This gives the upper bound of (6.3). As a consequence, $N_{\rho}(A)$ can be replaced by $\left|\mathbb{A}^{\rho}\right|$ in all the definitions of Section 2.2 as well as in (1.1).

We are now ready to prove the main theorem of the paper.

Proof of Theorem 1.1. We need to show that for any $\epsilon>0$, there exists a constant $C_{\epsilon}$ independent of $n$, such that for any set $A$ satisfying (1.1) we have that

$$
\mathbb{P}\left(\left|\mathcal{T}(n A)-\operatorname{dim}_{B}(A)(\log n+\log \log n)\right| \geq C_{\epsilon}\right) \leq \epsilon,
$$

for every $n$.

Fix $\epsilon>0$. For brevity, we define $A_{n}:=n A$ so that we can write $\mathbb{A}_{n}^{\rho}$ in place of $\Delta^{\rho}(n A)$. Our first step is to let

$$
\rho_{n}:=\frac{D}{\log \left|\mathbb{A}_{n}^{1}\right|},
$$

where $800 \log 2 \leq D<\infty$, and observe that by Lemma 4.1 part $a$ ) we have that $\rho_{n} \geq 800 \log 2\left(\log \left|\mathbb{A}_{n}^{\rho_{n}}\right|\right)^{-1}$. Then, according to Lemma 5.3 , the set $\mathbb{A}_{n}^{\rho_{n}}$ is good whenever $\left|\mathbb{A}_{n}^{1}\right|$ (and therefore also $\left|\mathbb{A}_{n}^{\rho_{n}}\right|$ ) is large enough. From now one we will simply assume that $n$ is so large that $\mathbb{A}_{n}^{\rho_{n}}$ is a good set. For reasons that will transpire, we shall further assume that $D$ is such that $c_{3} e^{-D / 600} \leq \epsilon / 4$, and (again for reasons that will become clear) we choose $C_{\epsilon} \geq 12 d D$ such that $e^{-C_{\epsilon} / 8} \leq \epsilon / 4$ and $\exp \left(-e^{C_{\epsilon} / 8}\right) \leq \epsilon / 4$.

Assume first that $A$ satisfies the assumption that for some $0<c_{A}<\infty$,

$$
\lim _{\rho \rightarrow 0} \rho^{\operatorname{dim}_{B}(A)}\left|\mathbb{A}^{\rho}\right|=c_{A} .
$$

This assumption is clearly stronger than (1.1), and the reason for this stronger assumption is to illustrate how the constant $c_{A}$ comes in to play (see also the remark after this proof). We will also let $\tilde{c}_{A}:=\operatorname{dim}_{B}(A)^{\operatorname{dim}_{B}(A)} c_{A}$.

It follows from (4.1) that for every $n \geq 1,\left|\mathbb{A}_{n}^{\rho_{n}}\right|=\left|n \mathbb{A}^{\rho_{n} / n}\right|=\left|\mathbb{A}^{\rho_{n} / n}\right|$ since enlarging a set does not change its cardinality. Therefore,

$$
\lim _{n \rightarrow \infty} \frac{\log \left|\mathbb{A}_{n}^{1}\right|}{\log n}=\lim _{n \rightarrow \infty} \frac{\log \left|\mathbb{A}^{1 / n}\right|}{\log n}=\operatorname{dim}_{B}(A),
$$


by the definition of $\operatorname{dim}_{B}(A)$ (see Section 2.2). We then get that

$$
\begin{gathered}
\left(\frac{D}{n \log n}\right)^{\operatorname{dim}_{B}(A)}\left|\mathbb{A}_{n}^{\rho_{n} \mid}=\left(\frac{\log \left|\mathbb{A}_{n}^{1}\right|}{\log n}\right)^{\operatorname{dim}_{B}(A)}\left(\frac{D}{n \log \left|\mathbb{A}_{n}^{1}\right|}\right)^{\operatorname{dim}_{B}(A)}\right| \mathbb{A}_{n}^{\rho_{n}} \mid \\
=\left(\frac{\log \left|\mathbb{A}_{n}^{1}\right|}{\log n}\right)^{\operatorname{dim}_{B}(A)}\left(\frac{\rho_{n}}{n}\right)^{\operatorname{dim}_{B}(A)}\left|\mathbb{A}^{\rho_{n} / n}\right| \rightarrow \operatorname{dim}_{B}(A)^{\operatorname{dim}_{B}(A)} c_{A}=\tilde{c}_{A},
\end{gathered}
$$

by (6.4) since $\rho_{n} / n \rightarrow 0$. Thus,

$$
\frac{1}{\tilde{c}_{A}}\left(\frac{D}{n \log n}\right)^{\operatorname{dim}_{B}(A)}\left|\mathbb{A}_{n}^{\rho_{n}}\right| \rightarrow 1 .
$$

Note further that $\left|\log D^{\operatorname{dim}_{B}(A)}\right| \leq|d \log D| \leq d D \leq C_{\epsilon} / 12$ by one of our assumptions on $C_{\epsilon}$. Thus, by (6.5) we have that

$$
\left|\log D^{\operatorname{dim}_{B}(A)}\right|+\left|\log \left(\frac{1}{\tilde{c}_{A}}\left(\frac{D}{n \log n}\right)^{\operatorname{dim}_{B}(A)}\left|\mathbb{A}_{n}^{\rho_{n}}\right|\right)\right| \leq C_{\epsilon} / 2,
$$

for every $n$ larger than some $N\left(D, C_{\epsilon}\right)$. Thus, for $n \geq N\left(D, C_{\epsilon}\right)$ we get

$$
\begin{aligned}
\mathbb{P}(\mid \mathcal{T} & \left.\left(A_{n}\right)-\operatorname{dim}_{B}(A)(\log n+\log \log n)-\log \tilde{c}_{A} \mid \geq C_{\epsilon}\right) \\
= & \mathbb{P}\left(\left|\mathcal{T}\left(A_{n}\right)-\log \right| \mathbb{A}_{n}^{\rho_{n}} \mid-\log D^{\operatorname{dim}_{B}(A)}\right. \\
& \left.\quad+\log \left(\frac{1}{\tilde{c}_{A}}\left(\frac{D}{n \log n}\right)^{\operatorname{dim}_{B}(A)}\left|\mathbb{A}_{n}^{\rho_{n}}\right|\right) \mid \geq C_{\epsilon}\right) \\
\leq & \mathbb{P}\left(\left|\mathcal{T}\left(A_{n}\right)-\log \right| \mathbb{A}_{n}^{\rho_{n}}|| \geq C_{\epsilon} / 2\right) \\
\leq & \mathbb{P}\left(\mathcal{T}\left(\mathbb{A}_{n}^{\rho_{n}}\right)-\log \left|\mathbb{A}_{n}^{\rho_{n}}\right| \leq-C_{\epsilon} / 2\right)+\mathbb{P}\left(\mathcal{T}_{w}^{\rho_{n}}\left(A_{n}\right)-\log \left|\mathbb{A}_{n}^{\rho_{n}}\right| \geq C_{\epsilon} / 2\right),
\end{aligned}
$$

where the last inequality follows since $\mathcal{T}\left(\mathbb{A}_{n}^{\rho_{n}}\right) \leq \mathcal{T}\left(A_{n}\right) \leq \mathcal{T}_{w}^{\rho_{n}}\left(A_{n}\right)$.

We shall now address the two probabilities of the right hand side of (6.6) separately. First, we can apply Theorem 5.7 with $\epsilon_{n}=\rho_{n} / 50$ to conclude that

$$
\left|\mathbb{P}\left(\mathcal{T}\left(\mathbb{A}_{n}^{\rho_{n}}\right)-\log \left|\mathbb{A}_{n}^{\rho_{n}}\right| \leq-C_{\epsilon} / 2\right)-\exp \left(-e^{C_{\epsilon} / 2}\right)\right| \leq c_{3}\left|\mathbb{A}_{n}^{\rho_{n}}\right|^{-\rho_{n} / 600}
$$

for every $n \geq N\left(D, C_{\epsilon}\right)$ (by perhaps making $N\left(D, C_{\epsilon}\right)$ even larger than before). Then, by using Lemma 4.1 part $a$ ) we see that

$$
\begin{aligned}
& \mathbb{P}\left(\mathcal{T}\left(\mathbb{A}_{n}^{\rho_{n}}\right)-\log \left|\mathbb{A}_{n}^{\rho_{n}}\right| \leq-C_{\epsilon} / 2\right) \leq \exp \left(-e^{C_{\epsilon} / 2}\right)+c_{3}\left|\mathbb{A}_{n}^{\rho_{n}}\right|^{-\rho_{n} / 600} \\
& \quad \leq \exp \left(-e^{C_{\epsilon} / 2}\right)+c_{3}\left|\mathbb{A}_{n}^{1}\right|^{-\rho_{n} / 600}=\exp \left(-e^{C_{\epsilon} / 2}\right)+c_{3} e^{-D / 600} \leq \epsilon / 2
\end{aligned}
$$

because of our choices of $D$ and $C_{\epsilon}$.

We now turn to the second term of the right hand side of (6.6). By Proposition 6.2 we have that $\left(1-\rho_{n}\right)^{d-1} \mathcal{T}_{w}^{\rho_{n}}\left(A_{n}\right)=\mathcal{T}\left(\Delta^{\rho_{n} /\left(1-\rho_{n}\right)}\left(\left(1-\rho_{n}\right)^{-1} A_{n}\right)\right)$. Furthermore, as above it follows from (4.1) that $\left|\Delta^{\rho_{n} /\left(1-\rho_{n}\right)}\left(\left(1-\rho_{n}\right)^{-1} A_{n}\right)\right|=$ $\left|\left(1-\rho_{n}\right)^{-1} \mathbb{A}_{n}^{\rho_{n}}\right|=\left|\mathbb{A}_{n}^{\rho_{n}}\right|$. We can therefore use Theorem 5.7 to conclude that

$$
\left|\mathbb{P}\left(\left(1-\rho_{n}\right)^{d-1} \mathcal{T}_{w}^{\rho_{n}}\left(A_{n}\right)-\log \left|\mathbb{A}_{n}^{\rho_{n}}\right| \leq z\right)-\exp \left(-e^{-z}\right)\right| \leq c_{3}\left|\mathbb{A}_{n}^{\rho_{n}}\right|^{-\rho_{n} / 600}
$$

for every $z \in \mathbb{R}^{d}$. Indeed, since we have that $\rho_{n} /\left(1-\rho_{n}\right)>\rho_{n} \geq D / \log \left|\mathbb{A}_{n}^{\rho_{n}}\right|$, the set $\Delta^{\rho_{n} /\left(1-\rho_{n}\right)}\left(\left(1-\rho_{n}\right)^{-1} A_{n}\right)$ is good and Theorem 5.7 can be applied for every $n \geq N\left(D, C_{\epsilon}\right)$.

By yet again picking $N\left(D, C_{\epsilon}\right)$ perhaps even larger than before, we have that $\rho_{n}=D / \log \left|\mathbb{A}_{n}^{1}\right| \leq 1-2^{-1 / d}$ for $n \geq N\left(D, C_{\epsilon}\right)$. We can then use the inequality 
$(1-x)^{-d+1} \leq 1+2 d x$ which holds for $0<x \leq 1-2^{-1 / d}$ to conclude that for such $n,\left(1-\rho_{n}\right)^{-(d-1)} \leq 1+2 d \rho_{n}$. Therefore, by Lemma 4.1 part $\left.c\right)$ we get that

$$
\begin{aligned}
& \log \left|\mathbb{A}_{n}^{\rho_{n}}\right|\left(\left(1-\rho_{n}\right)^{-(d-1)}-1\right) \leq \log \left(6^{d} \rho_{n}^{-d}\left|\mathbb{A}_{n}^{1}\right|\right) 2 d \rho_{n} \\
& =\left(d \log 6+\log \left|\mathbb{A}_{n}^{1}\right|+d \log \log \left|\mathbb{A}_{n}^{1}\right|-d \log \log D\right) 2 d \frac{D}{\log \left|\mathbb{A}_{n}^{1}\right|}
\end{aligned}
$$

Clearly, there exists an $N\left(D, C_{\epsilon}\right)$ perhaps even larger than before, such that for every $n \geq N\left(D, C_{\epsilon}\right)$,

$$
\log \left|\mathbb{A}_{n}^{\rho_{n}}\right|\left(\left(1-\rho_{n}\right)^{-(d-1)}-1\right) \leq 3 d D \leq C_{\epsilon} / 4,
$$

where we use the fact that $C_{\epsilon} \geq 12 d D$ by assumption.

Hence, for $n \geq N\left(D, C_{\epsilon}\right)$,

$$
\begin{aligned}
\mathbb{P}\left(\mathcal{T}_{w}^{\rho_{n}}\left(A_{n}\right)-\log \left|\mathbb{A}_{n}^{\rho_{n}}\right| \geq C_{\epsilon} / 2\right) \\
\quad=\mathbb{P}\left(\mathcal{T}_{w}^{\rho_{n}}\left(A_{n}\right)-\frac{\log \left|\mathbb{A}_{n}^{\rho_{n}}\right|}{\left(1-\rho_{n}\right)^{d-1}}+\frac{\log \left|\mathbb{A}_{n}^{\rho_{n}}\right|}{\left(1-\rho_{n}\right)^{d-1}}-\log \left|\mathbb{A}_{n}^{\rho_{n}}\right| \geq C_{\epsilon} / 2\right) \\
\quad \leq \mathbb{P}\left(\mathcal{T}_{w}^{\rho_{n}}\left(A_{n}\right)-\frac{\log \left|\mathbb{A}_{n}^{\rho_{n}}\right|}{\left(1-\rho_{n}\right)^{d-1}} \geq C_{\epsilon} / 4\right) \\
\quad=\mathbb{P}\left(\left(1-\rho_{n}\right)^{d-1} \mathcal{T}_{w}^{\rho_{n}}\left(A_{n}\right)-\log \left|\mathbb{A}_{n}^{\rho_{n}}\right| \geq\left(1-\rho_{n}\right)^{d-1} C_{\epsilon} / 4\right) \\
\quad \leq \mathbb{P}\left(\left(1-\rho_{n}\right)^{d-1} \mathcal{T}_{w}^{\rho_{n}}\left(A_{n}\right)-\log \left|\mathbb{A}_{n}^{\rho_{n}}\right| \geq C_{\epsilon} / 8\right)
\end{aligned}
$$

where the last inequality holds for every $n$ such that $\left(1-\rho_{n}\right)^{d-1} \geq 1 / 2$. This clearly holds for every $n \geq N\left(D, C_{\epsilon}\right)$ where $N\left(D, C_{\epsilon}\right)$ might be even larger than before.

We can now use (6.8) to see that

$$
\begin{aligned}
\mathbb{P}\left(\mathcal{T}_{w}^{\rho_{n}}\left(A_{n}\right)-\log \left|\mathbb{A}_{n}^{\rho_{n}}\right| \geq C_{\epsilon} / 2\right) \\
\quad \leq \mathbb{P}\left(\left(1-\rho_{n}\right)^{d-1} \mathcal{T}_{w}^{\rho_{n}}\left(A_{n}\right)-\log \left|\mathbb{A}_{n}^{\rho_{n}}\right| \geq C_{\epsilon} / 8\right) \\
\quad=1-\mathbb{P}\left(\left(1-\rho_{n}\right)^{d-1} \mathcal{T}_{w}^{\rho_{n}}\left(A_{n}\right)-\log \left|\mathbb{A}_{n}^{\rho_{n}}\right| \leq C_{\epsilon} / 8\right) \\
\quad \leq 1-\exp \left(-e^{-C_{\epsilon} / 8}\right)+c_{3}\left|\mathbb{A}_{n}^{\rho_{n}}\right|^{-\rho_{n} / 600} \leq e^{-C_{\epsilon} / 8}+c_{3} e^{-D / 600} \leq \epsilon / 2,
\end{aligned}
$$

much as when we dealt with the first term of the right hand side of (6.6).

Finally, combining (6.6), (6.7) and (6.9) we conclude that

$$
\left.\mathbb{P}\left(\mid \mathcal{T}\left(A_{n}\right)-\operatorname{dim}_{B}(A)(\log n+\log \log n)-\log \tilde{c}_{A}\right) \mid \geq C_{\epsilon}\right) \leq \epsilon
$$

for every $n \geq N\left(D, C_{\epsilon}\right)$. However it is now easy to see that (6.10) must in fact hold for every $n \geq 1$ by (perhaps) increasing $C_{\epsilon}$ even further.

The full statement (i.e. assuming (1.1) instead of (6.4)) is proved in a very similar way, and therefore we will only indicate the changes. Again we get that $\lim _{n \rightarrow \infty} \frac{\log \left|\mathbb{A}_{n}^{1}\right|}{\log n}=\operatorname{dim}_{B}(A)$ and the first change is that (6.5) is replaced by

$$
0<\liminf _{n \rightarrow \infty}\left(\frac{D}{n \log n}\right)^{\operatorname{dim}_{B}(A)}\left|\mathbb{A}_{n}^{\rho_{n}}\right| \leq \limsup _{n \rightarrow \infty}\left(\frac{D}{n \log n}\right)^{\operatorname{dim}_{B}(A)}\left|\mathbb{A}_{n}^{\rho_{n}}\right|<\infty .
$$

Then, one can pick $C_{\epsilon}$ perhaps even larger so that

$$
\left|\log D^{\operatorname{dim}_{B}(A)}\right|+\limsup _{n \rightarrow \infty}\left|\log \left(\left(\frac{D}{n \log n}\right)^{\operatorname{dim}_{B}(A)}\left|\mathbb{A}_{n}^{\rho_{n}}\right|\right)\right| \leq C_{\epsilon} / 3 .
$$


This can then be inserted into a slightly modified version of (6.6) in order to obtain the statement

$$
\begin{aligned}
\mathbb{P}\left(\left|\mathcal{T}\left(A_{n}\right)-\operatorname{dim}_{B}(A)(\log n+\log \log n)\right| \geq C_{\epsilon}\right) \\
\leq \mathbb{P}\left(\mathcal{T}\left(\mathbb{A}_{n}^{\rho_{n}}\right)-\log \left|\mathbb{A}_{n}^{\rho_{n}}\right| \leq-C_{\epsilon} / 2\right) \\
\quad+\mathbb{P}\left(\left(1-\rho_{n}\right)^{d-1} \mathcal{T}_{w}^{\rho_{n}}\left(A_{n}\right)-\log \left|\mathbb{A}_{n}^{\rho_{n}}\right| \geq C_{\epsilon} / 2\right),
\end{aligned}
$$

for every $n$ large enough, and then we can proceed as above.

Remark: Recall the remark after the statement of Theorem 1.1 where it is speculated that under strong enough regularity conditions $\left(\mathcal{T}(n A)-\operatorname{dim}_{B}(A)(\log n+\right.$ $\log \log n)+C)_{n \geq 1}$ might converge to a Gumbel distribution for some constant $C$. Then, (6.10) indicates that the constant $C$ might be the same as $-\log \tilde{c}_{A}=$ $-\operatorname{dim}_{B}(A) \log \operatorname{dim}_{B}(A)-\log c_{A}$. This is our reason for first proving Theorem 1.1 under the stronger assumption (6.4).

\section{Proof of Theorem 2.1}

In this section we shall prove our secondary result, i.e. Theorem 2.1.

Proof of Theorem 2.1. The proof is similar to the proof of Theorem 1.1 and so we shall be brief. One of the main differences is that here we let

$$
\rho_{n}:=\frac{\log \log n}{\log \left|\mathbb{A}_{n}^{1}\right|}
$$

where $A_{n}=n A$ as before. We remark that the choice of $\log \log n$ in the numerator is somewhat arbitrary. Indeed, any function that goes to infinity sufficiently slow as $n \rightarrow \infty$ would do. The purpose is to make sure that $\left|\mathbb{A}_{n}^{\rho_{n}}\right|^{-\rho_{n} / 600}$ vanishes in the limit and $\mathbb{A}_{n}^{\rho_{n}}$ is good.

Observe also that by (4.1),

$$
\liminf _{n \rightarrow \infty} \frac{\log \left|\mathbb{A}_{n}^{1}\right|}{\log n}=\liminf _{n \rightarrow \infty} \frac{\log \left|\mathbb{A}^{1 / n}\right|}{-\log (1 / n)} \geq \liminf _{\rho \rightarrow 0} \frac{\log \left|\mathbb{A}^{\rho}\right|}{-\log \rho}=\underline{\operatorname{dim}}_{B}(A) .
$$

Letting $\delta:=\left(\underline{\operatorname{dim}}_{B}(A)-\underline{\alpha}\right) / 2$ we then get that

$$
\begin{aligned}
& \left(\frac{\log \log n}{n \log n}\right)^{\operatorname{dim}_{B}(A)-\delta}\left|\mathbb{A}_{n}^{\rho_{n}}\right|
\end{aligned}
$$

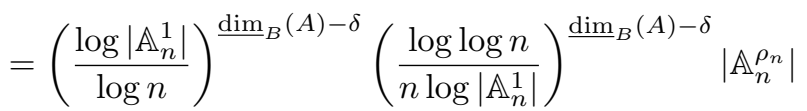

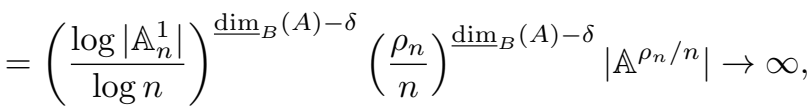

by the definition of $\underline{\operatorname{dim}}_{B}(A)$ (see Section 2.2). 
We then see that for $n$ larger than some $N$,

$$
\begin{aligned}
\mathbb{P}(\mathcal{T} & \left.\left(A_{n}\right)-\underline{\alpha} \log n \leq z\right) \\
= & \mathbb{P}\left(\mathcal{T}\left(A_{n}\right)-\log \left|\mathbb{A}_{n}^{\rho_{n}}\right|+\delta \log n+\left(\underline{\operatorname{dim}}_{B}(A)-\delta\right) \log \log n\right. \\
& \left.\quad-\log \left((\log \log n) \underline{\operatorname{dim}} \underline{B}_{B}(A)-\delta\right)+\log \left(\left(\frac{\log \log n}{n \log n}\right)^{\frac{\operatorname{dim}}{B}(A)-\delta}\left|\mathbb{A}_{n}^{\rho_{n}}\right|\right) \leq z\right) \\
\leq & \mathbb{P}\left(\mathcal{T}\left(A_{n}\right)-\log \left|\mathbb{A}_{n}^{\rho_{n}}\right|+\delta \log n \leq z\right) \\
\quad \leq & \mathbb{P}\left(\mathcal{T}\left(\mathbb{A}_{n}^{\rho_{n}}\right)-\log \left|\mathbb{A}_{n}^{\rho_{n}}\right| \leq z-\delta \log n\right) .
\end{aligned}
$$

where the last inequality follows since $\mathcal{T}\left(A_{n}\right) \geq \mathcal{T}\left(\mathbb{A}_{n}^{\rho_{n}}\right)$. Using Theorem 5.7 and Lemma 4.1 part $a$ ), we then get that

$$
\begin{aligned}
& \mathbb{P}\left(\mathcal{T}\left(A_{n}\right)-\underline{\alpha} \log n \leq z\right) \leq c_{3}\left|\mathbb{A}_{n}^{\rho_{n}}\right|^{-\rho_{n} / 600}+\exp \left(-e^{-z+\delta \log n}\right) \\
& \quad \leq c_{3}\left|\mathbb{A}_{n}^{1}\right|^{-\rho_{n} / 600}+\exp \left(-e^{-z} n^{\delta}\right)=c_{3} e^{-\log \log n / 600}+\exp \left(-e^{-z} n^{\delta}\right) \rightarrow 0 .
\end{aligned}
$$

The second statement is proved in the same way so we omit the proof.

\section{Applications}

The purpose of this section is two-fold. Firstly, we will demonstrate that any set containing a $d$-dimensional closed box satisfies (6.4), and so there are many examples of sets for which our main result apply. Presumably, results such as Proposition 8.1 are well known, even though we could not find a reference for this exact statement. Secondly we will consider examples of sets where $\operatorname{dim}_{B}(A)<d$ and see what our main results imply for those sets.

Proposition 8.1. Let $A=[0,1]^{d}$. Then,

$$
0<\lim _{\rho \rightarrow 0} \rho^{d}\left|\mathbb{A}^{\rho}\right|<\infty,
$$

and in particular the limit exists. It follows that any bounded set $A$ such that $[x, x+\delta]^{d} \subset A$ for some $x \in \mathbb{R}^{d}$ and $\delta>0$, must satisfy (1.1).

Proof. Let $c>0$ and note that $A=[0,1]^{d}$ is contained in the union of $\left(\left\lceil(c \rho)^{-1}\right\rceil\right)^{d}$ translates of the set $A_{c \rho}=[0, c \rho]^{d}$. It follows from (4.1) that $\left|\mathbb{A}_{c \rho}^{\rho}\right|=\left|\mathbb{A}^{\rho /(c \rho)}\right|=$ $\left|\mathbb{A}^{1 / c}\right|$. Then, by using Lemma 4.1 part $\left.b\right)$, we get that

$$
\left|\mathbb{A}^{\rho}\right| \leq\left(\left\lceil(c \rho)^{-1}\right\rceil\right)^{d}\left|\mathbb{A}_{c \rho}^{\rho}\right| \leq\left((c \rho)^{-1}+1\right)^{d}\left|\mathbb{A}^{1 / c}\right| \text {. }
$$

Therefore,

$$
\limsup _{\rho \rightarrow 0} \rho^{d}\left|\mathbb{A}^{\rho}\right| \leq \limsup _{\rho \rightarrow 0}\left(c^{-1}+\rho\right)^{d}\left|\mathbb{A}^{1 / c}\right|=c^{-d}\left|\mathbb{A}^{1 / c}\right|,
$$

and so

$$
\limsup _{\rho \rightarrow 0} \rho^{d}\left|\mathbb{A}^{\rho}\right| \leq \liminf _{c \rightarrow \infty} c^{-d}\left|\mathbb{A}^{1 / c}\right|=\liminf _{\rho \rightarrow 0} \rho^{d}\left|\mathbb{A}^{\rho}\right| .
$$

This proves that the limit exists.

We then observe that (8.1) immediately implies that the limit is finite. Furthermore, we must have that

$$
\left|\mathbb{A}^{\rho}\right| \geq\left|A \cap\left(\rho \mathbb{Z}^{d}\right)\right| \geq\left(\rho^{-1}-1\right)^{d},
$$

and so $\lim _{\rho \rightarrow 0} \rho^{d}\left|\mathbb{A}^{\rho}\right| \geq 1$. 
We now turn to the second statement. To that end, $A \subset \mathbb{R}^{d}$ is now any bounded set that includes some box $B=[x, x+\delta]^{d}$. Thus,

$$
\liminf _{\rho \rightarrow 0} \rho^{d}\left|\mathbb{A}^{\rho}\right| \geq \liminf _{\rho \rightarrow 0} \rho^{d}\left|\mathbb{B}^{\rho}\right|>0,
$$

by the first statement of the proposition. Similarly, for large enough $\Delta$, we let $D=[y, y+\Delta]^{d} \supset A$ and then we find that

$$
\limsup _{\rho \rightarrow 0} \rho^{d}\left|\mathbb{A}^{\rho}\right| \leq \limsup _{\rho \rightarrow 0} \rho^{d}\left|\mathbb{D}^{\rho}\right|<\infty .
$$

Example 8.2. Proposition 8.1 lets us apply Theorem 1.1 to the set $[0,1]^{d}$. Informally, we then know that $\mathcal{T}\left(n[0,1]^{d}\right)$ will be of order $d(\log n+\log \log n)$.

A discrete analogue of $n[0,1]^{d}$ might be taken to be $\mathbb{D}_{n}=[0, n-1]^{d} \cap \mathbb{Z}^{d}$. However, Theorem 1.2 implies that $\mathcal{T}\left(\mathbb{D}_{n}\right)$ will be of order $\log \left|\mathbb{D}_{n}\right|=d \log n$. Thus, the sequence $\left(\mathcal{T}\left(\mathbb{D}_{n}\right)\right)_{n \geq 1}$ behaves differently from the sequence $\left(\mathcal{T}\left(n[0,1]^{d}\right)\right)_{n \geq 1}$ in that the first is "missing" the extra factor $d \log \log n$. We point out that the results of Belius (2012) are similar to the ones for $\left(\mathbb{D}_{n}\right)_{n \geq 1}$, while the results of Janson (1986) are similar to when the ones for $\left(n[0,1]^{d}\right)_{n \geq 1}$. This seems to indicate that the extra factor $d \log \log n$ arises from the covered sets being non-discrete rather then from the fact that we are using unbounded cylinders to perform our covering.

Of course, it is important to point out that the sequence $\left(\mathbb{D}_{n}\right)_{n \geq 1}$ is not obtained by starting with some $B$ and then multiplying it by $n$.

Example 8.3. In this example we consider a two-dimensional Cantor set. Since this is a well known set we shall be somewhat informal in its description (see Falconer, 2014 Example 4.3 for details when $d=1$ ). We also remark that one can easily generalize this set into higher dimensions. We start with the unit box $F_{0}=[0,1]^{2}$, and in our first step we delete everything except the corner cubes of side length $1 / 3$. Thus, we let $F_{1}=[0,1 / 3]^{2} \cup([0,1 / 3] \times[2 / 3,1]) \cup([2 / 3,1] \times[0,1 / 3]) \cup[2 / 3,1]^{2}$. We then continue by repeating the exact same procedure on a smaller scale within each of the four retained sub-boxes in order to obtain $F_{2}$. Continuing, we obtain a sequence $F_{k} \supset F_{k+1} \supset \cdots$ where $F_{k}$ consists of $4^{k}$ boxes of side length $3^{-k}$. We then define

$$
F:=\cap_{k=1}^{\infty} F_{k},
$$

and it is easy to check that $\operatorname{dim}_{B}(F)=\frac{\log 4}{\log 3}$.

It is not hard to see that $\mathbb{F}^{3^{-k}}$ must consist of the union of all four corner points of the sub boxes of $\mathbb{F}_{k}$. Indeed, each sub box has side length $3^{-k}$ so that the distance between two adjacent corner points is exactly $3^{-k}$. Therefore, each sub box cannot contain more than these four points. Thus, $\left|\mathbb{F}^{3^{-k}}\right|=4^{k}$ and we see that

$$
\lim _{k \rightarrow \infty}\left(3^{-k}\right)^{\frac{\log 4}{\log 3}}\left|\mathbb{F}^{3^{-k}}\right|=\lim _{k \rightarrow \infty} 4^{-k} 44^{k}=4,
$$

and by interpolating we get that

$$
1=\liminf _{\rho \rightarrow 0} \rho^{\frac{\log 4}{\log 3}}\left|\mathbb{F}^{\rho}\right| \leq \limsup _{\rho \rightarrow 0} \rho^{\frac{\log 4}{\log 3}}\left|\mathbb{F}^{\rho}\right|=4 .
$$


Therefore, Theorem 1.1 can be applied to show that the sequence

$$
\left(\mathcal{T}(n F)-\frac{\log 4}{\log 3}(\log n+\log \log n)\right)_{n \geq 1}
$$

is tight. Informally, we can say that the cover time $\mathcal{T}(n F)$ will be of order $\frac{\log 4}{\log 3}(\log n+\log \log n)$ with some fluctuations.

Example 8.4. Here we let $A=[0,1]^{k} \times\{0\}^{d-k} \subset \mathbb{R}^{d}$. As in Proposition 8.1 it is easy to verify that Theorem 1.1 is applicable. Then, we conclude that for any dimensions $d$, the sequence

$$
(\mathcal{T}(n A)-k(\log n+\log \log n))_{n \geq 1}
$$

is tight. However, the constants in our results are allowed to depend on $d$, so it is possible that a stronger or at least different result can be obtained by letting $d \rightarrow \infty$ at the same time as $n \rightarrow \infty$. However, we choose not to pursue this here.

Example 8.5. Our last example will be of a sequence of finite sets. To that end, consider $\mathbb{I}_{n}$ consisting of $\frac{n \log n}{\log \log n}$ equidistant points on the interval $[0, n]$. Then, let $\mathbb{B}_{n}=\mathbb{I}_{n}^{d}=\mathbb{I}_{n} \times \cdots \times \mathbb{I}_{n}$. Clearly,

$$
\operatorname{Sep}\left(\mathbb{B}_{n}\right) \log \left|\mathbb{B}_{n}\right|=\frac{n}{\frac{n \log n}{\log \log n}} \log \left(\frac{n \log n}{\log \log n}\right)^{d}=d \log \log n+O\left((\log n)^{-1 / 2}\right) \rightarrow \infty,
$$

so that Theorem 1.2 tells us that $\mathcal{T}\left(\mathbb{B}_{n}\right)-\log \left|\mathbb{B}_{n}\right|=\mathcal{T}\left(\mathbb{B}_{n}\right)-d(\log n+\log \log n-$ $\log \log \log n$ ) converges to a Gumbel distributed random variable.

Note that if $\mathbb{I}_{n}$ would instead consist of $n \log n$ points, then Theorem 1.2 would not be applicable. However, estimates can be obtained by using Theorem 5.7 on approximations of the set.

Acknowledgement. The authors would like to thank S. Janson, J. Tykesson and J. Steif for many useful comments and suggestions. We would also like to thank the anonymous referees for their input which has much improved the paper.

\section{References}

D. Belius. Cover levels and random interlacements. Ann. Appl. Probab. 22 (2), 522-540 (2012). MR2953562.

H. Biermé and A. Estrade. Covering the whole space with Poisson random balls. ALEA Lat. Am. J. Probab. Math. Stat. 9, 213-229 (2012). MR2923191.

E. I. Broman, J. Jonasson and J. Tykesson. The existence phase transition for two Poisson random fractal models. Electron. Commun. Probab. 22, Paper No. 21, 8 (2017). MR3635694.

A. Dvoretzky. On covering a circle by randomly placed arcs. Proc. Nat. Acad. Sci. U.S.A. 42, 199-203 (1956). MR79365.

K. Falconer. Fractal geometry. John Wiley \& Sons, Ltd., Chichester, third edition (2014). ISBN 978-1-119-94239-9. MR3236784.

H. Federer. Geometric measure theory. Die Grundlehren der mathematischen Wissenschaften, Band 153. Springer-Verlag New York Inc., New York (1969). MR0257325. 
S. Janson. Random coverings of the circle with arcs of random lengths. In Probability and mathematical statistics, pages 62-73. Uppsala Univ., Uppsala (1983). MR727128.

S. Janson. Random coverings in several dimensions. Acta Math. 156 (1-2), 83-118 (1986). MR822331.

S. Li. Concise formulas for the area and volume of a hyperspherical cap. Asian J. Math. Stat. 4 (1), 66-70 (2011). MR2813331.

R. Schneider and W. Weil. Stochastic and integral geometry. Probability and its Applications (New York). Springer-Verlag, Berlin (2008). ISBN 978-3-540-788584. MR2455326.

L. A. Shepp. Covering the line with random intervals. Z. Wahrscheinlichkeitstheorie und Verw. Gebiete 23, 163-170 (1972). MR322923.

A. F. Siegel and L. Holst. Covering the circle with random arcs of random sizes. $J$. Appl. Probab. 19 (2), 373-381 (1982). MR649974.

A.-S. Sznitman. Vacant set of random interlacements and percolation. Ann. of Math. (2) 171 (3), 2039-2087 (2010). MR2680403.

J. Tykesson and D. Windisch. Percolation in the vacant set of Poisson cylinders. Probab. Theory Related Fields 154 (1-2), 165-191 (2012). MR2981421. 